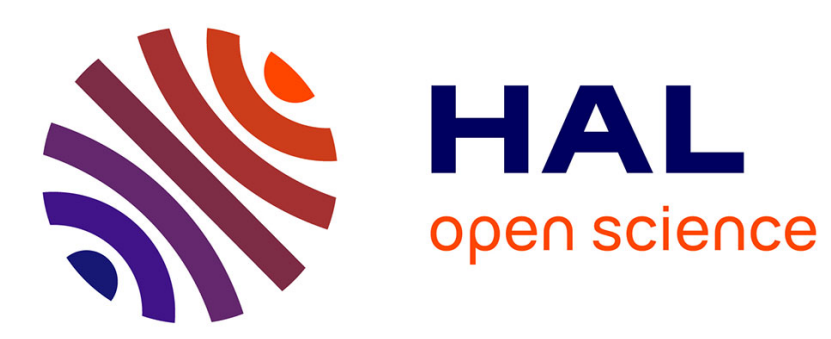

\title{
A discontinuous Galerkin scheme for front propagation with obstacles
}

\author{
Olivier Bokanowski, Yingda Cheng, Chi-Wang Shu
}

\section{To cite this version:}

Olivier Bokanowski, Yingda Cheng, Chi-Wang Shu. A discontinuous Galerkin scheme for front propagation with obstacles. 2011. hal-00653532v1

\section{HAL Id: hal-00653532 \\ https://hal.inria.fr/hal-00653532v1}

Preprint submitted on 19 Dec 2011 (v1), last revised 12 Oct 2012 (v2)

HAL is a multi-disciplinary open access archive for the deposit and dissemination of scientific research documents, whether they are published or not. The documents may come from teaching and research institutions in France or abroad, or from public or private research centers.
L'archive ouverte pluridisciplinaire HAL, est destinée au dépôt et à la diffusion de documents scientifiques de niveau recherche, publiés ou non, émanant des établissements d'enseignement et de recherche français ou étrangers, des laboratoires publics ou privés. 


\title{
A DISCONTINUOUS GALERKIN SCHEME FOR FRONT PROPAGATION WITH OBSTACLES
}

\author{
OLIVIER BOKANOWSKI, YINGDA CHENG, AND CHI-WANG SHU
}

\begin{abstract}
We are interested in front propagation problems in the presence of obstacles. We extend a previous work (Bokanowski, Cheng and Shu [6]), to propose a simple and direct discontinuous Galerkin (DG) method adapted to such front propagation problems. We follow the formulation of Bokanowski et al. [8], leading to a level set formulation driven by $\min \left(u_{t}+H(x, \nabla u), u-g(x)\right)=0$, where $g(x)$ is an obstacle function. The DG scheme is motivated by the variational formulation when the Hamiltonian $H$ is a linear function of $\nabla u$, corresponding to linear convection problems in presence of obstacles. The scheme is then generalized to nonlinear equations, written in an explicit form. Stability analysis are performed for the linear case with Euler forward, a Heun scheme and a Runge-Kutta third order time discretization using the technique proposed in Zhang and Shu [22]. Several numerical examples are provided to demonstrate the robustness of the method. Finally, a narrow band approach is considered in order to reduce the computational cost.
\end{abstract}

\section{INTRODUCTION}

We are interested in numerical schemes for front propagation problems that comes from deterministic attainability problems, or optimal control problems.

When there is no constraint, the front propagation problems we study can be modeled by the following Hamilton-Jacobi (HJ) equation

$$
\begin{aligned}
& u_{t}+H(x, \nabla u)=0, \quad x \in \mathbb{R}^{d}, \quad t>0, \\
& u(0, x)=u_{0}(x), \quad x \in \mathbb{R}^{d} .
\end{aligned}
$$

where $u=u(t, x)$ is a real valued function, and $H$ will be made more precise in Section 2 . A discontinuous Galerkin (DG) scheme for such equations has been proposed in [6].

In the present work, we shall consider the following equation

$$
\min \left(u_{t}+H(x, \nabla u), u-g(x)\right)=0, \quad x \in \mathbb{R}^{d}, \quad t>0,
$$

together with an initial condition (1b). Here $g(x)$ will be called the "obstacle function", and (2) will be referred as the "obstacle equation". This function $g$ is motivated by the modeling of constraints in the original control problem (the region $g(x) \leq 0$ will correspond to the set of constraints).

Taking constraints in optimal control problems is not an obvious task. In the work of Soner [20], or in Frankowska et al. [14], a HJ equation characterizing the value of such

Date: Nov. 2010, submitted to Numerisch Math.

Key words and phrases. Hamilton-Jacobi-Bellman equations; discontinuous Galerkin methods; level sets; front propagation; obstacle problems; stability;

Research supported by NSF grant DMS-1016001.

Research supported by ARO grant W911NF-08-1-0520 and NSF grant DMS-0809086. 
problems are obtained, under different (yet restrictive) assumptions on the boundary of the set of constraints (related to "local controllability" assumptions on the problem). In Cardaliaguet et al. [10], a general characterization using a nonsmooth analysis framework (viability theory) was obtained, but the development of approximation schemes in this nonsmooth setting remains a challenging task $[19,11,9]$.

Recently it was remarked in Bokanowski et al. [8] that (2) could be used to code the reachable sets of optimal control problems by using $u$ as a level set function, and without making any particular assumption on the boundary of the set of constraints. This approach was furthermore numerically tested with finite difference schemes. It can be used to recover various objects such as the minimal time function.

Our aim here is to make use of the potential of DG schemes on such problems. The Runge-Kutta discontinuous Galerkin (RKDG) methods were originally devised to solve the conservation laws, see [13] and the reference within. DG methods have many attractive features, such as flexibility with complicated geometries, ability to achieve high order accuracy using compact stencils and efficient parallel implementations. In $[15,17]$, DG methods were developed to solve the HJ equations based on the conservation law system satisfied by the derivatives of the solution. The design of direct DG solvers for HJ equations, however, is not an easy task, because the viscosity solution does not have the weak formulation where the design of most DG schemes rely on. In [12], a DG method for directly solving the Hamilton-Jacobi equation was developed. Extensive numerical tests have been performed for equations with linear and convex nonlinear Hamiltonians. This scheme has provable stability and error estimates for linear equations and demonstrates good convergence to the viscosity solutions for nonlinear equations. Recently, some other direct solvers have also been proposed, such as the central DG scheme [18] and the local DG scheme [21].

This paper is based on our previous work [6], where a DG scheme for HJ equations arising from front propagation was considered using the direct solver in [12]. The method was shown to have very good long time behavior, without the need of "reinitialization" because of the high precision of the method. It can resolve thin targets very well and has potentials for parallel implementation on unstructured grids.

In this paper, we propose several fully discrete and explicit DG methods for (2). These include schemes with Euler forward, second (RK2) and third order (RK3) explicit Runge Kutta time discretization. To do so, we shall use a natural and equivalent variational formulation that is already known for obstacle problems in a special case (see (3) below). Compared to traditional finite element methods for such problems, the DG scheme proposed does not require solving a nonlinear equation at each time step. Rather, the obstacles are incorporated by a simple projection step given explicitly through a comparison with the obstacle functions at Gaussian quadrature points. The scheme is shown to be compact and high order in smooth regions.

We derive stability estimates for these fully discrete schemes, in the particular case where $H(x, \nabla u)$ is linear in $\nabla u$ (hereafter mentioned as the "linear" case, although the equation is nonlinear because of the obstacle term), leading to an equation of the form

$$
\min \left(u_{t}+f(x) \cdot \nabla u, u-g(x)\right)=0, \quad x \in \mathbb{R}^{d} .
$$

Our strategy will be mainly to use stability estimates of Zhang and Shu [22] for linear equations, and extend them to the obstacle case (under a CFL condition such as $\tau / h \leq$ 
const, where $\tau$ and $h$ are the time and space steps respectively, for the RK2 and RK3 schemes).

The plan of the paper is as follows. In Section 2 we present the variational formulation and discussion for the obstacle equations. We show that this formulation is equivalent to (3) in the "linear" case. In Section 3, we introduce the scheme and its motivation. Section 4 contains a proof of the stability results for the fully discrete schemes. Finally in Section 5 we give several one dimensional and two dimensional numerical examples illustrating the validity of our approach. We observe the designed high order accuracy in the smooth part of the solution and good resolution to the singularities (discontinuities in the solution derivatives). A narrow band method is also tested in the last example, showing potential speed up of the numerical method.

\section{The obstacle EQUATION}

2.1. Motivation for the obstacle equation. First, let us consider the following HJ equation (no obstacle):

$$
\begin{aligned}
& \varphi_{t}+\max _{\alpha \in \mathcal{A}}(f(\mathbf{x}, \alpha) \cdot \nabla \varphi)=0, \quad t \geq 0, \quad \mathbf{x} \in \mathbb{R}^{d} \\
& \varphi(0, \mathbf{x})=\varphi^{0}(\mathbf{x}), \quad \mathbf{x} \in \mathbb{R}^{d}
\end{aligned}
$$

where $\mathcal{A}$ is a non-empty compact set. This is a special case of (1) when $H(x, \nabla \varphi)=$ $\max _{\alpha \in \mathcal{A}}(f(\mathbf{x}, \alpha) \cdot \nabla \varphi)$. We assume that $f: \mathbb{R}^{d} \times \mathcal{A} \rightarrow \mathbb{R}^{d}$ is globally Lipschitz continuous in both variables $\mathbf{x}$ and $\alpha$. Then equation (4) comes from the following optimal control problem:

$$
\varphi(t, \mathbf{x}):=\inf \left\{\varphi^{0}\left(y_{\mathbf{x}}^{\alpha}(-t)\right), \alpha \in L^{\infty}((0, t), \mathcal{A})\right\},
$$

where $y=y_{\mathrm{x}}^{\alpha}$ is the absolutely continuous solution of $\dot{y}(\theta)=f(y(\theta), \alpha(\theta))$ for almost every $\theta \in[-t ; 0]$ and with $y(0)=\mathbf{x}$ (see for instance Bardi and Capuzzo-Dolcetta [2]).

This model is motivated by a general type of non-isotropic front propagation problems. By front propagation we mean that we focus on the computation of the 0-level set $\{\mathbf{x}, \varphi(t, \mathbf{x})=0\}$. Then, Eq. (4) models the expansion of the negative region $\mathcal{R}_{t}:=$ $\{\mathbf{x}, \varphi(t, \mathbf{x}) \leq 0\}$ with maximal possible speed, where $f_{\alpha}$ is the set of possible dynamics $[2]$.

Suppose now that we consider the same front propagation problem in the presence of obstacles. Let $K$ be a given closed set of $\mathbb{R}^{d}$ (the set of constraints). It represents the "allowed" region for the trajectory $y(\theta)$. by

We look for the region $\mathcal{R}_{t}$ (as well as its front $\partial \mathcal{R}_{t}$ ) where now $\varphi$ is naturally defined

(6) $\varphi(t, \mathbf{x}):=\inf \left\{\varphi^{0}\left(y_{\mathbf{x}}^{\alpha}(-t)\right), \alpha \in L^{\infty}((-t, 0), \mathcal{A})\right.$ and $\left.y_{\mathbf{x}}^{\alpha}(\theta) \in K, \forall \theta \in(-t, 0)\right\}$.

In the case there is no trajectory satisfying the constraint $y_{\mathbf{x}}^{\alpha}(\theta) \in K, \forall \theta$, we define $\varphi(t, x)=\infty$ (we could also choose a large numerical value). It is not easy to define, in the general case, a PDE for (6), in particular because $\varphi$ is no more continuous but only lower semi continuous (however, see [7] for some tentative approaches).

To deal with this problem, the approach proposed in [8] is the following. We first set a Lipschitz continuous function $g(x)$ such that

$$
g(x) \leq 0 \Leftrightarrow x \in K
$$


(for instance $g(x)=d_{K}(x)$, the signed distance to $K$ ). Then we consider the following unconstrained problem:

$$
u(t, x):=\inf \left\{\max \left(\varphi^{0}\left(y_{x}^{\alpha}(-t)\right), \max _{\theta \in(-t, 0)} g\left(y_{x}^{\alpha}(\theta)\right)\right), \alpha \in L^{\infty}((-t, 0), \mathcal{A})\right\} .
$$

It is easy to see that $u$ satisfies

$$
\mathcal{R}_{t}=\{x, u(t, x) \leq 0\} .
$$

Furthermore we have an obstacle-like equation for $u$ :

$$
\begin{aligned}
& \min \left(u_{t}+H(x, \nabla u), u-g(x)\right)=0, \quad t>0, x \in \mathbb{R}^{d}, \\
& u(0, x)=u_{0}(x):=\max \left(\varphi^{0}(x), g(x)\right), \quad x \in \mathbb{R}^{d}
\end{aligned}
$$

where $H(x, p):=\max _{a \in \mathcal{A}} f(x, \alpha) \cdot p$. The above equation should be understood in the viscosity sense. More precisely, if $\varphi^{0}$ is Lipschitz continuous, there exists a unique Lipschitz continuous function $u$ which is a viscosity solution of (8), and it is given by (7). The advantage of (8) is that it gives a continuous setting approach for the front $\partial \mathcal{R}_{t}$.

Before going on, we notice that there are other known equations with continuous solution for the obstacle problem.

Remark 2.1 (Penalization approach for the obstacle problem). We consider the problem to reach a target $\mathcal{C}$. It is known from [16] (see also [8]), that if we consider $\bar{\varphi}^{0} \geq 0$ such that

$$
\bar{\varphi}^{0}(x)=0 \Leftrightarrow x \in \mathcal{C}
$$

(such as $\bar{\varphi}^{0}(x)=d(x, \mathcal{C})$ ), and an obstacle function $\bar{g}$ such that

$$
\bar{g}(x)=0 \Leftrightarrow x \in K,
$$

(for instance, $\bar{g}(x)=d(x, K)$ ), then the function defined by

$$
\bar{u}(t, x):=\min \left\{\bar{\varphi}^{0}\left(y_{x}^{\alpha}(-t)\right)+\int_{0}^{t} \bar{g}\left(y_{x}^{\alpha}(-\theta)\right) d \theta, \alpha \in L^{\infty}([-t, 0] ; \mathcal{A})\right\}
$$

will satisfy $\bar{u}(t, x) \geq 0$ and also

$$
\mathcal{R}_{t}=\{x, \bar{u}(t, x)=0\} .
$$

Now, by standard arguments (dynamic programming principle), we can show that $\bar{u}$ satisfies the following PDE in the viscosity sense:

$$
\begin{aligned}
\bar{u}_{t}+H(x, \nabla \bar{u})-\bar{g}(x) & =0, \quad t>0, x \in \mathbb{R}^{d} \\
\bar{u}(0, x) & =\bar{\varphi}^{0}(x), \quad x \in \mathbb{R}^{d} .
\end{aligned}
$$

The numerical drawback of this approach is that the solution tends to increase values indefinitely with time, when $x \notin K$. Also $\bar{u}$ will always have at most Lipschitz continuous regularity and will not change sign (it goes from zero to positive values) near the front $\partial \mathcal{R}_{t}$, whereas in the obstacle approach we can have more regularity and in general $u$ will change signs at the front. We have included a numerical example (see Remark 5.1) for comparison with our approach. 
2.2. A variational formulation. Let us assume that the data $\varphi_{0}$ and the function $g$ are Lipschitz continuous, so that the solution of (8) is also Lipschitz continuous (see [3,8], this can be obtained directly by the formula (7)). We can also consider that $u_{0}=\varphi^{0} \geq g$ a.e. (otherwise it suffices to replace $\varphi^{0}$ by $\max \left(\varphi^{0}, g\right)$ ). Hence the solution $u$ is almost everywhere differentiable by Rademacher's Theorem: the equation is valid in the classical sense at almost every (a.e.) point. This means $u_{t}+H(x, \nabla u(x)) \geq 0, u \geq g(x)$ and one of the two term is zero: $\left(u_{t}+H(x, \nabla u(x))\right)(u(t, x)-g(x))=0$. In particular, if $($,$) denotes$ the $L^{2}$ scalar product, then

$$
\left(u_{t}+H(., \nabla u), u-g\right)=0 .
$$

At this point we assume that the scalar product is finite, so that (11) makes sense (we could also consider a periodic setting on a box $\Omega$, with corresponding $L^{2}$ scalar product).

Now, let $V^{g}$ be defined by

$$
V^{g}=\left\{v \in L^{2}\left(\mathbb{R}^{d}\right), v(x) \geq g(x) \text { a.e. }\right\}
$$

(or, in the periodic setting to avoid technical difficulties, $V^{g}$ would be the set of functions $v \in L_{\text {loc }}^{2}\left(\mathbb{R}^{d}\right)$ with periodic boundary conditions such that $v \geq g$ a.e.) Let $v$ be in $V^{g}$. Using the previous identity, we obtain

$$
\left(u_{t}+H(., \nabla u), v-u\right)=\left(u_{t}+H(., \nabla u), v-g\right) \geq 0
$$

Hence we obtain a variational formulation for (8): to find $u$ such that, for a.e. $t>0$,

$$
u(t, .) \geq g, \quad \text { and } \quad\left(u_{t}+H(., \nabla u), v-u\right) \geq 0, \quad \forall v \in V^{g},
$$

and

$$
u(0, .)=u_{0} .
$$

Conversely, assume that $u$ is Lipschitz continuous and satisfies (13) for a.e. $t>0$. For any Lebesgue's point $a$ of the function $h:=u_{t}(t,)+.H(., \nabla u(t,)$.$) , we have h(a) \geq 0$. (It suffices to take $v_{\varepsilon}(x):=u(t, x)+\frac{1}{\epsilon^{d}} \delta\left(\frac{x-a}{\epsilon}\right)$ where $\delta$ is any function with compact support such that $\delta \geq 0$ and $\int_{\mathbb{R}^{d}} \delta(x) d x=1$. Then letting $\epsilon \rightarrow 0$ we obtain $0 \leq\left(h, v_{\epsilon}-u\right)=$ $\int h(a+\epsilon y) \delta(y) d y \rightarrow h(a)$.) On the other hand, $u(t, x) \geq g(x)$ a.e. by assumption. Finally, taking the test function $v(x)=g(x)$ we obtain $(h, u-g)=0$. Since both terms $h$ and $u-g$ are non-negative, this implies $h(x)(u(t, x)-g(x))=0$, a.e. We thus obtain, for a.e. $t>0$,

$$
\min \left(u_{t}+H(x, \nabla u), u-g(x)\right)=0, \quad \text { a.e. } x \in \mathbb{R}^{d} .
$$

In general this does not prove that $u$ is the value function given by (7). In the following we give one particular case where this is true.

2.3. Existence and uniqueness result in the case when $H$ is linear. Here we consider the particular "linear" case, when $H(x, \nabla u) \equiv f(x) \cdot \nabla u$, and the equation is thus

$$
\begin{aligned}
& \min \left(u_{t}+f(x) \cdot \nabla u, u-g(x)\right)=0, \quad x \in \mathbb{R}^{d}, t>0, \\
& u(0, x)=u_{0}(x), \quad x \in \mathbb{R}^{d} .
\end{aligned}
$$

(here we assume that $u_{0}(x) \geq g(x)$ a.e. as before) For this problem, we remark that there is existence and uniqueness of the solution $u$ of the variational formulation with $u$ 
Lipschitz continuous (and derivatives $u_{t}$ and $\nabla u$ taken in the distributional sense). Here this variational formulation becomes: find $u$ Lipschitz continuous such that

(16a) $u(t,) \geq g,$.$\quad and \left(u_{t}+f(x) \cdot \nabla u, v-u\right) \geq 0, \quad \forall v \in V^{g}$, a.e. $t>0$,

and

$$
u(0, .)=u_{0}
$$

First let us notice that such a problem was already considered for instance in [4] for minimal time problems and in the particular case when $g(x) \equiv 0$ (see also [5]). To show the existence of a solution of (15), one can consider for instance formula (7) and proceed as in the previous section to check that it is a solution. To prove uniqueness, let us consider two solutions $u_{1}$ and $u_{2}$ of (16), and let $w=u_{2}-u_{1}$. Then considering (16a) for $u=u_{1}$ with test function $v=u_{2}(t,$.$) , and the same (16a) for u=u_{2}$ with test function $v=u_{1}(t,$.$) ,$ and by taking the difference, we obtain

$$
\left(w_{t}+f \cdot \nabla w, w\right) \leq 0
$$

Hence, using Stoke's formula,

$$
\begin{aligned}
\frac{d}{d t}(w, w) & \leq 2(-w, f \cdot \nabla w)=\int f(x) \cdot \nabla\left(w^{2}\right) d x \\
& =-\int \operatorname{div}(f(x)) w^{2} d x \\
& \leq C(w, w),
\end{aligned}
$$

for some constant $C \geq 0$. Then using a Gronwall Lemma we deduce that $(w, w) \leq$ $e^{C t}(w(0), w(0))=0$, thus $w \equiv 0$ and $u_{2} \equiv u_{1}$.

Hence we have shown the equivalence between the "min" formulation of the obstacle problem (15) (or (7)), and the variational formulation (16).

Note that the affine case, where $H(x, \nabla u) \equiv f(x) \cdot \nabla u+q(x)$ for some functions $f$ and $q$, could be treated in the same way.

\section{A DG Finite ElEMENT METHOd FOR the obstacle PROBlems}

In this section, we will formulate a DG finite element method for the obstacle problem (2). To motivate our scheme, we will first consider (2) when the Hamiltonian is a linear function of $\nabla u$.

3.1. A one-dimensional model problem with a linear Hamiltonian. We consider the following one-dimensional model problem on the interval $I$ with periodic boundary conditions:

$$
\min \left(u_{t}+u_{x}, u-g(x)\right)=0 .
$$

The domain $I=\bigcup_{1 \leq j \leq N} I_{j}$, where $I_{j}=\left[x_{j-\frac{1}{2}}, x_{j+\frac{1}{2}}\right] . h_{j}=x_{j+\frac{1}{2}}-x_{j-\frac{1}{2}}$, and $h=\max _{j} h_{j}$. The finite element space $V_{h}:=\left\{v_{h},\left.v_{h}\right|_{I_{j}} \in \mathbb{P}^{k}, \forall j\right\}$, where $\mathbb{P}^{k}$ is the set of polynomials of degree less than or equal to $k$.

For the unconstrained problem $u_{t}+u_{x}=0$, the standard DG scheme with forward Euler time discretization can be written as: to find $u_{h}^{n+1} \in V_{h}$, such that

$$
\left(u_{h}^{n+1}-u_{h}^{n}, v_{h}\right)-\tau \mathcal{H}\left(u_{h}^{n}, v_{h}\right)=0, \quad \forall v_{h} \in V_{h} .
$$


Here $\tau$ is the time step, $(\phi, \varphi)=\int_{I} \phi \varphi_{x} d x$ and

$$
\mathcal{H}(\phi, \varphi)=\int_{I} \phi \varphi_{x} d x+\sum_{j} \phi_{j+\frac{1}{2}}^{-}[\varphi]_{j+\frac{1}{2}}
$$

where $[\varphi]=\varphi^{+}-\varphi^{-}$denotes the jump of the function $\varphi$ at cell interface.

Now we propose a DG scheme for (17) with forward Euler discretization as follows: to find $u_{h}^{n+1} \in V_{h}^{g}$, such that

$$
\left(u_{h}^{n+1}-u_{h}^{n}, v_{h}-u_{h}^{n+1}\right)-\tau \mathcal{H}\left(u_{h}^{n}, v_{h}-u_{h}^{n+1}\right) \geq 0, \quad \forall v_{h} \in V_{h}^{g} .
$$

The space $V_{h}^{g}:=\left\{v_{h} \in V_{h},, v_{h}\left(x_{\alpha}^{j}\right) \geq g\left(x_{\alpha}^{j}\right), \forall j, \alpha\right\}$, where $\left\{x_{\alpha}^{j}\right\}_{\alpha=1}^{k+1}$ are the $(k+1)$ Gaussian points on cell $I_{j}, j=1, \ldots, N$. Clearly, (20) can be viewed as a discrete version of (13). The choice for the finite element space $V_{h}^{g}$ and the Gaussian points will be elaborated more later in this section.

The scheme (20) is now well defined but hard to implement because of the inequality and the non-standard space $V_{h}^{g}$. To address this difficulty, the main idea of our approach is to rewrite (20) in an equivalent form that is closely related to the unconstrained scheme (18). Now we define $\varphi_{\alpha}^{j}(\cdot)$ to be Lagrange polynomials on $I_{j}$, such that $\varphi_{\alpha}^{j}\left(x_{\beta}^{j}\right)=\delta_{\alpha \beta}$. Then $\left.u_{h}^{n}\right|_{I_{j}}=\sum_{\alpha=1}^{k+1} u_{\alpha}^{n, j} \varphi_{\alpha}^{j}(x)$, where $u_{\alpha}^{n, j}$ is the point value of $u_{h}^{n}$ at $x_{\alpha}^{j}$. Define the vector $U^{n, j}=\left\{u_{1}^{n, j}, u_{2}^{n, j}, \ldots, u_{k+1}^{n, j}\right\}$, and $U^{n}=\left\{U^{n, 1}, U^{n, 2}, \ldots, U^{n, N}\right\}$ then (18) can be written equivalently as:

$$
\forall V \in \mathbb{R}^{k+1}, \quad\left(U^{n+1, j} M-U^{n, j} A-U^{n, j-1} B, V\right)=0
$$

with $(V, W):=\sum_{\alpha=1}^{k+1} V_{j} W_{j} . M$ is the mass matrix and $M_{\alpha \beta}=\left(\varphi_{\alpha}^{j}, \varphi_{\beta}^{j}\right)=w_{\alpha} \delta_{\alpha \beta}$ where $w_{\alpha}>0$ is the Gaussian weight. $A$ and $B$ are $(k+1) \times(k+1)$ matrices. Since $(21)$ holds for any $V \in \mathbb{R}^{k+1}$, we obtain

$$
U^{n+1, j} M-U^{n, j} A-U^{n, j-1} B=0,
$$

which can be equivalently written as

$$
U^{n+1}=F\left(U^{n}\right) .
$$

Now, we consider the scheme (20). Denote $G_{\alpha}^{j}=g\left(x_{\alpha}^{j}\right)$, then

$$
V_{h}^{g}=\left\{\varphi \in V_{h} \text {, such that } \varphi\left(x_{\alpha}^{j}\right) \geq G_{\alpha}^{j} \text { for all } j, \alpha\right\} .
$$

We define a vector $V \geq G$ if all the element of $V$ is greater than the corresponding element in $G$. (20) can be written in the vector form as: to find $U^{n+1} \geq G$ such that

$$
\forall V \geq G, \quad\left(U^{n+1, j} M-U^{n, j} A-U^{n, j-1} B, V-U^{n+1, j}\right) \geq 0
$$

Using the fact that $M$ is positive and diagonal, taking $V=U^{n+1}$ except on interval $I_{j}$ and for an index $\alpha$, we obtain

$$
\forall V_{\alpha}^{j} \geq G_{\alpha}^{j}, \quad\left(w_{j} U_{\alpha}^{n+1, j}-\left(U^{n, j} A+U^{n, j-1} B\right)_{\alpha}^{j}\right)\left(V_{\alpha}^{j}-U_{\alpha}^{n+1, j}\right) \geq 0 .
$$

Hence $w_{j} U_{\alpha}^{n+1, j}-\left(U^{n, j} A+U^{n, j-1} B\right)_{\alpha}^{j} \geq 0$, for all $\alpha, j$, by taking $V_{\alpha}^{j}$ large enough. Since $U^{n+1} \geq G$, we have

$$
\left(w_{j} U_{\alpha}^{n+1, j}-\left(U^{n, j} A+U^{n, j-1} B\right)_{\alpha}^{j}\right)\left(G_{\alpha}^{j}-U_{\alpha}^{n+1, j}\right) \leq 0 .
$$


On the other hand, let $V_{\alpha}^{j}=G_{\alpha}^{j}$ in (23),

$$
\left(w_{j} U_{\alpha}^{n+1, j}-\left(U^{n, j} A+U^{n, j-1} B\right)_{\alpha}^{j}\right)\left(G_{\alpha}^{j}-U_{\alpha}^{n+1, j}\right) \geq 0 .
$$

Hence,

$$
\left(w_{j} U_{\alpha}^{n+1, j}-\left(U^{n, j} A+U^{n, j-1} B\right)_{\alpha}^{j}\right)\left(G_{\alpha}^{j}-U_{\alpha}^{n+1, j}\right)=0 .
$$

This implies $U_{\alpha}^{n+1, j} \geq \frac{1}{w_{j}}\left(U^{n, j} A+U^{n, j-1} B\right)_{\alpha}^{j}=F\left(U^{n}\right)_{\alpha}^{j}$ and $U_{\alpha}^{n+1, j} \geq G_{\alpha}^{j}$ and one of the two inequalities must be an equality. Thus,

$$
U_{\alpha}^{n+1, j}=\max \left(F\left(U^{n}\right)_{\alpha}^{j}, G_{\alpha}^{j}\right) .
$$

Conversely this last equation implies also (22). In conclusion, the DG scheme in (20) is equivalent to (24), i.e. we can

(1) compute $\tilde{u}_{h}^{n+1}$ from solving the unconstrained problem $u_{t}+u_{x}=0$ by the standard DG method, namely to solve

$$
\left(\tilde{u}_{h}^{n+1}-u_{h}^{n}, v_{h}\right)-\tau \mathcal{H}\left(u_{h}^{n}, v_{h}\right)=0, \quad \forall v_{h} \in V_{h}
$$

(2) "The Projection Step". Take the maximum of $\tilde{u}_{h}^{n+1}$ and $g(x)$ at the Gaussian points,

$$
u_{\alpha}^{n+1, j}:=\max \left(\left(\tilde{u}_{h}\right)_{\alpha}^{n+1, j}, G_{\alpha}^{j}\right) .
$$

Then recover $u_{h}^{n+1}$ from those point values.

Remark 3.1. We can clearly see that the construction of the equivalent scheme (24) relies heavily on the fact that $M$ is a diagonal matrix. In general, if $M$ is not diagonal, we can not deduce a simple form as in (24). This justifies the choice of Gaussian points in the finite element space $V_{h}^{g}$.

Remark 3.2. The variational approach for the obstacle problem is known and has been used for instance for finance options (American options), see [1]. Usually, working with continuous elements leads to a non trivial non linear equation to solve at each time step (even for the "explicit euler" scheme), of the form $\min (M x-b, x-g)=0$ (componentwise). The use of the DG scheme for the obstacle problem is new (to our best knowledge), as well as the fact that it simplifies significantly the implementation by taking the maximum at the Gaussian points.

3.2. The DG scheme for general obstacle problems. In this subsection, we will formulate a DG finite element method for the general obstacle problems (2). Without loss of generality, we focus on the case of $d=2$, i.e., two-dimensional problems.

3.2.1. The $D G$ scheme for the unconstrained problem in piecewise $Q^{k}$ space. First, we will review a DG scheme for the unconstrained problem (1) proposed in our previous work [6]. We restrict our attention to the case of Cartesian grids. The main feature of the scheme below is the choice of the finite element space $V_{h}$ as piecewise $Q^{k}=\mathbb{P}^{k} \otimes \mathbb{P}^{k}$ space and a new entropy fix criteria adapted for this space. We will motivate this choice in the next subsection.

The two-dimensional Hamilton-Jacobi equation is given by

$$
\varphi_{t}+H\left(\varphi_{x}, \varphi_{y}, x, y\right)=0, \quad \varphi(x, y, 0)=\varphi^{0}(x, y)
$$


Suppose the domain of computation is $[a, b] \times[c, d]$. We shall use rectangular meshes defined as

$$
a=x_{\frac{1}{2}}<x_{\frac{3}{2}}<\ldots<x_{N_{x}+\frac{1}{2}}=b, \quad c=y_{\frac{1}{2}}<y_{\frac{3}{2}}<\ldots<y_{N_{y}+\frac{1}{2}}=d
$$

and

$$
\begin{aligned}
& I_{i, j}=\left[x_{i-\frac{1}{2}}, x_{i+\frac{1}{2}}\right] \times\left[y_{j-\frac{1}{2}}, y_{j+\frac{1}{2}}\right], \quad J_{i}=\left[x_{i-1 / 2}, x_{i+1 / 2}\right], \quad K_{j}=\left[y_{j-1 / 2}, y_{j+1 / 2}\right] \\
& J_{i+1 / 2}=\left[x_{i}, x_{i+1}\right], \quad K_{j+1 / 2}=\left[y_{j}, y_{j+1}\right], \quad i=1, \ldots N_{x}, \quad j=1, \ldots N_{y},
\end{aligned}
$$

where $x_{i}=\frac{1}{2}\left(x_{i-\frac{1}{2}}+x_{i+\frac{1}{2}}\right)$, and $y_{j}=\frac{1}{2}\left(y_{j-1 / 2}+y_{j+1 / 2}\right)$. We define the approximation space as

$$
V_{h}=\left\{v:\left.v\right|_{I_{i, j}} \in Q^{k}\left(I_{i, j}\right), i=1, \ldots N_{x}, \quad j=1, \ldots N_{y}\right\}
$$

where $Q^{k}\left(I_{i, j}\right)$ denotes all polynomials of degree at most $k$ in $x$ and $y$ on $I_{i, j}$.

Let us denote $H_{1}=\frac{\partial H}{\partial \varphi_{x}}$ and $H_{2}=\frac{\partial H}{\partial \varphi_{y}}$. In the cell $I_{i, j}$ we define

$$
\begin{aligned}
& H_{1, \text { min }}^{\varphi_{h}, i}(y):=\min \left(0, \min _{x \in J_{i+1 / 2}} H_{1}\left(\partial_{x} \varphi_{h}(x, y), \overline{\partial_{y} \varphi_{h}}(x, y), x_{i+1 / 2}, y\right)\right) \\
& H_{1, \text { max }}^{\varphi_{h}, i}(y):=\max \left(0, \max _{x \in J_{i-1 / 2}} H_{1}\left(\partial_{x} \varphi_{h}(x, y), \overline{\partial_{y} \varphi_{h}}(x, y), x_{i-1 / 2}, y\right)\right)
\end{aligned}
$$

and

$$
\begin{aligned}
& H_{2, \text { min }}^{\varphi_{h}, j}(x):=\min \left(0, \min _{y \in K_{j+1 / 2}} H_{2}\left(\overline{\partial_{x} \varphi_{h}}(x, y), \partial_{y} \varphi_{h}(x, y), x, y_{j+1 / 2}\right)\right) \\
& H_{2, \text { max }}^{\varphi_{h}, j}(x):=\max \left(0, \max _{y \in K_{j-1 / 2}} H_{2}\left(\overline{\partial_{x} \varphi_{h}}(x, y), \partial_{y} \varphi_{h}(x, y), x, y_{j-1 / 2}\right)\right)
\end{aligned}
$$

where we have used the notations

$$
\overline{\partial_{x} \varphi_{h}}=\frac{1}{2}\left(\left(\partial_{x} \varphi_{h}\right)^{+}+\left(\partial_{x} \varphi_{h}\right)^{-}\right), \quad \overline{\partial_{y} \varphi_{h}}=\frac{1}{2}\left(\left(\partial_{y} \varphi_{h}\right)^{+}+\left(\partial_{y} \varphi_{h}\right)^{-}\right) .
$$

Here and below, the superscript + is used to denote the right (in $\mathrm{x}$ direction) or top (in $\mathrm{y}$ direction) limit of the function, whereas, the superscript - is used to denote the left (in $\mathrm{x}$ direction) or bottom (in y direction) limit of the function.

Then the scheme introduced in [12] is: find $\varphi_{h}(x, t) \in V_{h}^{k}$, such that

$$
\begin{aligned}
0=\int_{I_{i, j}}\left(\partial_{t} \varphi_{h}(x, y, t)+H(\right. & \left.\left.\partial_{x} \varphi_{h}(x, y, t), \partial_{y} \varphi_{h}(x, y, t), x, y\right)\right) v_{h}(x, y) d x d y \\
& +\int_{K_{j}} H_{1, \min }^{\varphi_{h}, i}(y)\left[\varphi_{h}\right]\left(x_{i+\frac{1}{2}}, y\right) v_{h}\left(x_{i+\frac{1}{2}}^{-}, y\right) d y \\
& +\int_{K_{j}} H_{1, \max }^{\varphi_{h}, i}(y)\left[\varphi_{h}\right]\left(x_{i-\frac{1}{2}}, y\right) v_{h}\left(x_{i-\frac{1}{2}}^{+}, y\right) d y \\
& +\int_{J_{i}} H_{2, \min }^{\varphi_{h}, j}(x)\left[\varphi_{h}\right]\left(x, y_{j+\frac{1}{2}}\right) v_{h}\left(x, y_{j+\frac{1}{2}}^{-}\right) d x \\
& +\int_{J_{i}} H_{2, \max }^{\varphi_{h}, j}(x)\left[\varphi_{h}\right]\left(x, y_{j-\frac{1}{2}}\right) v_{h}\left(x, y_{j-\frac{1}{2}}^{+}\right) d x
\end{aligned}
$$


holds for any $v_{h} \in V_{h}^{k}$, where we have also denoted $\left[\varphi_{h}\right]\left(x_{i+\frac{1}{2}}, y\right):=\varphi_{h}\left(x_{i+\frac{1}{2}}^{+}, y\right)-$ $\varphi_{h}\left(x_{i+\frac{1}{2}}^{-}, y\right)$ and $\left[\varphi_{h}\right]\left(x, y_{j+\frac{1}{2}}\right):=\varphi_{h}\left(x, y_{j+\frac{1}{2}}^{+}\right)-\varphi_{h}\left(x, y_{j+\frac{1}{2}}^{-}\right)$.

Because the solution is discontinuous at interfaces of cells, reconstructions are needed. Along the normal direction of the interface, we would use the $L^{2}$ reconstructed information of the partial derivatives as in the one dimensional case. Tangential to the interface, the average of the partial derivatives from the two neighboring cells is used. The reconstruction process is described in [12].

For general nonlinear equations, a suitable entropy correction is necessary to guarantee the stability of the scheme. The criteria for the violation of entropy condition are simple and are described below. We say the entropy condition is violated at $\left(x_{i \pm \frac{1}{2}}, y\right)$, if

$$
H_{1}\left(\partial_{x} \varphi_{h}\left(x_{i \pm \frac{1}{2}}^{-}, y\right), \partial_{y} \varphi_{h}\left(x_{i \pm \frac{1}{2}}^{-}, y\right), x_{i \pm \frac{1}{2}}, y\right)<0
$$

and

$$
H_{1}\left(\partial_{x} \varphi_{h}\left(x_{i \pm \frac{1}{2}}^{+}, y\right), \partial_{y} \varphi_{h}\left(x_{i \pm \frac{1}{2}}^{+}, y\right), x_{i \pm \frac{1}{2}}, y\right)>0 .
$$

Similarly, the entropy condition is violated at $\left(x, y_{j \pm \frac{1}{2}}\right)$, if

$$
H_{2}\left(\partial_{x} \varphi_{h}\left(x, y_{j \pm \frac{1}{2}}^{-}\right), \partial_{y} \varphi_{h}\left(x, y_{j \pm \frac{1}{2}}^{-}\right), x, y_{j \pm \frac{1}{2}}\right)<0
$$

and

$$
H_{2}\left(\partial_{x} \varphi_{h}\left(x, y_{j \pm \frac{1}{2}}^{+}\right), \partial_{y} \varphi_{h}\left(x, y_{j \pm \frac{1}{2}}^{+}\right), x, y_{j \pm \frac{1}{2}}\right)>0 .
$$

The entropy correction described below will degenerate to at most second order when the entropy correction is performed. In order to avoid unnecessary corrections, we add one additional constraint. We will only do the above entropy fix if

$$
\left.H\left(\partial_{x} \varphi_{h}\left(x_{i}, y_{j}\right), \partial_{y} \varphi_{h}\left(x_{i}, y_{j}\right), x_{i}, y_{j}\right)\right)>\epsilon,
$$

where $\epsilon$ is a small number which is taken as $10^{-3}$ in our numerical experiments.

Below we outline an improved version of entropy fix over [6]. For cell $I_{i, j}$, if the entropy condition is violated, we cast it into four categories.

(1) If the entropy condition is violated at the right boundary points $\left(x_{i+\frac{1}{2}}, y\right)$, and $\varphi_{h}\left(x_{i+\frac{1}{2}}^{+}, y\right)$ is not in the interval between $\varphi_{h}\left(x_{i}, y\right)$ and $\varphi_{h}\left(x_{i+1}, y\right)$, then replace the term $\left[\varphi_{h}\right]\left(x_{i+\frac{1}{2}}, y\right)$ in $(29)$ by $\left(\varphi_{h}\left(x_{i+1}, y\right)+\varphi_{h}\left(x_{i}, y\right)\right) / 2-\varphi_{h}\left(x_{i+\frac{1}{2}}^{-}, y\right)$. In practice, we only need to do it for the $y$ values that are located at the Gaussian points.

(2) If the entropy condition is violated at the left boundary points $\left(x_{i-\frac{1}{2}}, y\right)$, and $\varphi_{h}\left(x_{i-\frac{1}{2}}^{-}, y\right)$ is not in the interval between $\varphi_{h}\left(x_{i-1}, y\right)$ and $\varphi_{h}\left(x_{i}, y\right)$, then replace the term $\left[\varphi_{h}\right]\left(x_{i-\frac{1}{2}}, y\right)$ in $(29)$ by $\varphi_{h}\left(x_{i-\frac{1}{2}}^{+}, y\right)-\left(\varphi_{h}\left(x_{i-1}, y\right)+\varphi_{h}\left(x_{i}, y\right)\right) / 2$. In practice, we only need to do it for the $y$ values that are located at the Gaussian points.

(3) If the entropy condition is violated at the top boundary points $\left(x, y_{j+\frac{1}{2}}\right)$, and $\varphi_{h}\left(x, y_{j+\frac{1}{2}}^{+}\right)$is not in the interval between $\varphi_{h}\left(x, y_{j}\right)$ and $\varphi_{h}\left(x, y_{j+1}\right)$, then replace the term $\left[\varphi_{h}\right]\left(x, y_{j+\frac{1}{2}}\right)$ in $(29)$ by $\left(\varphi_{h}\left(x, y_{j+1}\right)+\varphi_{h}\left(x, y_{j}\right)\right) / 2-\varphi_{h}\left(x, y_{j+\frac{1}{2}}^{-}\right)$. In 
practice, we only need to do it for the $x$ values that are located at the Gaussian points.

(4) If the entropy condition is violated at the bottom boundary points $\left(x, y_{j-\frac{1}{2}}\right)$, and $\varphi_{h}\left(x, y_{j-\frac{1}{2}}^{-}\right)$is not in the interval between $\varphi_{h}\left(x, y_{j-1}\right)$ and $\varphi_{h}\left(x, y_{j}\right)$, then replace the term $\left[\varphi_{h}\right]\left(x, y_{j-\frac{1}{2}}\right)$ in $(29)$ by $\varphi_{h}\left(x, y_{j-\frac{1}{2}}^{+}\right)-\left(\varphi_{h}\left(x, y_{j}\right)+\varphi_{h}\left(x, y_{j-1}\right)\right) / 2$. In practice, we only need to do it for the $x$ values that are located at the Gaussian points.

Note that the main difference of the above scheme compared to the one in $[6]$ is that we no longer look for the entropy violation in terms of the four cell boundaries. Instead, we enforce the criteria at each point along the boundaries. It turns out this scheme will be more stable especially for the $Q^{k}$ polynomial space.

3.2.2. The DG scheme for the general obstacle problems. We propose the following DG scheme for (2) inspired by the discussion in Subsection 3.1.

At each time step from $t^{n}$ to $t^{n+1}$,

(1) compute $\tilde{u}_{h}^{n+1}$ from $u_{h}^{n}$ by solving the unconstrained problem (1) using the DG method described in the previous subsection. The time discretization can be chosen as total variation diminishing (TVD) Runge-Kutta method of arbitrary order.

(2) "The Projection Step". Take the maximum of $\tilde{u}_{h}^{n+1}$ and $g(x)$ at Gaussian points $\mathbf{x}_{\alpha, \beta}^{i, j}, \alpha, \beta=1, \ldots, k+1, i=1, \ldots, N_{x}, j=1, \ldots, N_{y}$,

$$
u_{\alpha, \beta}^{n+1, i, j}:=\max \left(\left(\tilde{u}_{h}\right)_{\alpha, \beta}^{n+1, i, j}, G_{\alpha, \beta}^{i, j}\right) .
$$

Then recover $u_{h}^{n+1}$ from those point values. Here the notation $\mathbf{x}_{\alpha, \beta}^{i, j}=\left(x_{\alpha}^{i}, y_{\beta}^{j}\right)$ denotes the tensor product of one-dimensional Gaussian points. The choice of piecewise $Q^{k}$ space made those points natural and the mass matrix diagonal.

For the case of linear Hamiltonians, our scheme can be written in a compact form just as in (20) in Subsection 3.1. For example, the second order Runge-Kutta (RK2) scheme for $u_{t}+u_{x}=0$ can be written as: to find $u_{h}^{n, 1}, \tilde{u}_{h}^{n+1} \in V_{h}$, such that

$$
\begin{aligned}
& \left(u_{h}^{n, 1}-u_{h}^{n}, v_{h}\right)-\tau \mathcal{H}\left(u_{h}^{n}, v_{h}\right)=0, \quad \forall v_{h} \in V_{h} \\
& \left(\tilde{u}_{h}^{n+1}-\frac{1}{2} u_{h}^{n}-\frac{1}{2} u_{h}^{n, 1}, v_{h}\right)-\frac{\tau}{2} \mathcal{H}\left(u_{h}^{n, 1}, v_{h}\right)=0, \quad \forall v_{h} \in V_{h} .
\end{aligned}
$$

Then the RK2 scheme for (17) is: to find $u_{h}^{n, 1} \in V_{h}$ and $u_{h}^{n+1} \in V_{h}^{g}$, such that

$$
\begin{aligned}
& \left(u_{h}^{n, 1}-u_{h}^{n}, v_{h}\right)-\tau \mathcal{H}\left(u_{h}^{n}, v_{h}\right)=0, \quad \forall v_{h} \in V_{h} \\
& \left(u_{h}^{n+1}-\frac{1}{2} u_{h}^{n}-\frac{1}{2} u_{h}^{n, 1}, v_{h}-u_{h}^{n+1}\right)-\frac{\tau}{2} \mathcal{H}\left(u_{h}^{n, 1}, v_{h}-u_{h}^{n+1}\right) \geq 0, \quad \forall v_{h} \in V_{h}^{g} .
\end{aligned}
$$


The third order TVD RK scheme for $u_{t}+u_{x}=0$ is: to find $u_{h}^{n, 1}, u_{h}^{n, 2}, \tilde{u}_{h}^{n+1} \in V_{h}$, such that

$$
\begin{aligned}
& \left(u_{h}^{n, 1}-u_{h}^{n}, v_{h}\right)-\tau \mathcal{H}\left(u_{h}^{n}, v_{h}\right)=0, \quad \forall v_{h} \in V_{h} \\
& \left(u_{h}^{n, 2}-\frac{3}{4} u_{h}^{n}-\frac{1}{4} u_{h}^{n, 1}, v_{h}\right)-\frac{\tau}{4} \mathcal{H}\left(u_{h}^{n, 1}, v_{h}\right)=0, \quad \forall v_{h} \in V_{h}, \\
& \left(\tilde{u}_{h}^{n+1}-\frac{1}{3} u_{h}^{n}-\frac{2}{3} u_{h}^{n, 2}, v_{h}\right)-\frac{2 \tau}{3} \mathcal{H}\left(u_{h}^{n, 2}, v_{h}\right)=0, \quad \forall v_{h} \in V_{h} .
\end{aligned}
$$

And the RK3 scheme for (17) is: to find $u_{h}^{n, 1}, u_{h}^{n, 2} \in V_{h}$ and $u_{h}^{n+1} \in V_{h}^{g}$, such that

$$
\begin{aligned}
& \left(u_{h}^{n, 1}-u_{h}^{n}, v_{h}\right)-\tau \mathcal{H}\left(u_{h}^{n}, v_{h}\right)=0, \quad \forall v_{h} \in V_{h} \\
& \left(u_{h}^{n, 2}-\frac{3}{4} u_{h}^{n}-\frac{1}{4} u_{h}^{n, 1}, v_{h}\right)-\frac{\tau}{4} \mathcal{H}\left(u_{h}^{n, 1}, v_{h}\right)=0, \quad \forall v_{h} \in V_{h}, \\
& \left(u_{h}^{n+1}-\frac{1}{3} u_{h}^{n}-\frac{2}{3} u_{h}^{n, 2}, v_{h}-u_{h}^{n+1}\right)-\frac{2 \tau}{3} \mathcal{H}\left(u_{h}^{n, 2}, v_{h}-u_{h}^{n+1}\right) \geq 0, \quad \forall v_{h} \in V_{h}^{g} .
\end{aligned}
$$

The compact formulations above will be used for the stability analysis in Section 4 .

\section{Stability analysis}

In this section, we will consider the stability of our proposed scheme for problems with linear Hamiltonians and periodic boundary condition as described in Subsection 3.1. Because the proposed scheme does not have a semi-discrete version in its nature, we will analyze the scheme with associated time discretization. We focus on one-dimensional equations, although the proof can be readily generalized to the multi-dimensional cases.

We begin by collecting some of the properties of the operator $\mathcal{H}$ in (19) that was reported in [22]. Below, $\|\cdot\|$ denotes the $L^{2}$ norm on $I$, and $\|\cdot\|_{\Gamma_{h}}$ denotes the $L^{2}$ norm on the boundaries, i.e.

$$
\|\eta\|_{\Gamma_{h}}=\left[\sum_{1 \leq j \leq N}\left(\eta_{j+\frac{1}{2}}^{+}\right)^{2}+\left(\eta_{j+\frac{1}{2}}^{-}\right)^{2}\right]^{1 / 2} .
$$

We will also invoke the inverse inequalities for functions $v_{h}$ in $V_{h}$,

$$
\left\|\left(v_{h}\right)_{x}\right\| \leq \mu h^{-1}\left\|v_{h}\right\|, \quad\left\|v_{h}\right\|_{\Gamma_{h}} \leq \mu h^{-1 / 2}\left\|v_{h}\right\|,
$$

where $\mu$ only depends on the polynomial order $k$. The following lemma about the operator $\mathcal{H}$ was reported in [22].

Lemma 4.1. For any $\phi, \varphi \in V_{h}$, we have

$$
|\mathcal{H}(\phi, \varphi)| \leq \mu h^{-1}\|\phi\| \cdot\|\varphi\|,
$$

where $\mu$ only depends on the polynomial order $k$. For any $\phi, \varphi \in H^{1, h}(I)$, we have

$$
\begin{aligned}
& \mathcal{H}(\phi, \varphi)+\mathcal{H}(\varphi, \phi)=-\sum_{1 \leq j \leq N}[\phi]_{j+\frac{1}{2}}[\varphi]_{j+\frac{1}{2}} \\
& \mathcal{H}(\phi, \phi)=-\frac{1}{2} \sum_{1 \leq j \leq N}[\phi]_{j+\frac{1}{2}}^{2} .
\end{aligned}
$$


4.1. Stability for the forward Euler scheme. In this subsection, we prove the stability for the forward Euler scheme (20). It is well known that DG schemes for conservation laws will not be stable with forward Euler time stepping and $k \geq 1$ when $\tau=O(h)$. In the theorem below, we will assume $\tau=O\left(h^{2}\right)$.

Below, $g_{h}$ denotes the unique interpolation function of $g$ in space $V_{h}$, i.e. $g_{h} \in V_{h}$ and $g_{h}\left(x_{\alpha}^{j}\right)=g\left(x_{\alpha}^{j}\right)$. Since $g$ is Lipschitz continuous, we can prove that there exists constant $C_{g}$, such that

$$
\|g\|_{H^{1}} \leq C_{g}
$$

and

$$
\left\|\epsilon_{g}\right\|+h\left\|\left(\epsilon_{g}\right)_{x}\right\|+\sqrt{h}\left\|\epsilon_{g}\right\|_{\Gamma_{h}} \leq C_{g} h
$$

where $\epsilon_{g}=g-g_{h}$.

Theorem 4.1. Under the CFL condition $\tau \leq C_{1} h^{2}$ and when $h$ is small enough, the scheme (20) is stable, i.e.

$$
\left\|u_{h}^{n}-g_{h}\right\| \leq e^{C_{0} t}|| u_{h}^{0}-g_{h} \|,
$$

and

$$
\left\|u_{h}^{n}\right\| \leq e^{C_{0} t}\left\|u_{h}^{0}-g_{h}\right\|+\left\|g_{h}\right\|,
$$

where the constant $C_{0}=C_{1}^{2} \mu^{2}+2 C_{g}(\mu+1)$.

Proof. We have

$$
\begin{aligned}
\left\|u_{h}^{n+1}-u_{h}^{n}\right\|^{2}+\left\|u_{h}^{n+1}-g_{h}\right\|^{2}-\left\|u_{h}^{n}-g_{h}\right\|^{2} \\
=2\left(u_{h}^{n+1}-u_{h}^{n}, u_{h}^{n+1}-g_{h}\right) \leq 2 \tau \mathcal{H}\left(u_{h}^{n}, u_{h}^{n+1}-g_{h}\right) \\
=2 \tau \mathcal{H}\left(u_{h}^{n+1}-g_{h}, u_{h}^{n+1}-g_{h}\right)+2 \tau \mathcal{H}\left(g_{h}, u_{h}^{n+1}-g_{h}\right) \\
\quad-2 \tau \mathcal{H}\left(u_{h}^{n+1}-u_{h}^{n}, u_{h}^{n+1}-g_{h}\right),
\end{aligned}
$$

where the second line follows from the scheme (20) by choosing the test function to be $g_{h}$. Using (32), we have

$$
\mathcal{H}\left(u_{h}^{n+1}-g_{h}, u_{h}^{n+1}-g_{h}\right)=-\frac{1}{2} \sum_{1 \leq j \leq N}\left[u_{h}^{n+1}-g_{h}\right]^{2} .
$$

To estimate the second term, we use

$$
\mathcal{H}\left(g_{h}, u_{h}^{n+1}-g_{h}\right)=\mathcal{H}\left(g, u_{h}^{n+1}-g_{h}\right)-\mathcal{H}\left(\epsilon_{g}, u_{h}^{n+1}-g_{h}\right)
$$

and since $g$ is Lipschitz continuous, by (31),

$$
\begin{aligned}
& \mathcal{H}\left(g, u_{h}^{n+1}-g_{h}\right)=-\mathcal{H}\left(u_{h}^{n+1}-g_{h}, g\right) \\
& =\int_{I}\left(u_{h}^{n+1}-g_{h}\right) g_{x} d x \leq\left\|u_{h}^{n+1}-g_{h}\right\| \cdot\left\|g_{x}\right\| \leq C_{g}\left\|u_{h}^{n+1}-g_{h}\right\| .
\end{aligned}
$$

By the same argument in the proof of Lemma 4.1 in [22] and inverse inequalities with (33), we obtain

$$
\begin{aligned}
\left|\mathcal{H}\left(\epsilon_{g}, u_{h}^{n+1}-g_{h}\right)\right| & \leq\left\|\epsilon_{g}\right\| \cdot\left\|\left(u_{h}^{n+1}-g_{h}\right)_{x}\right\|+\sqrt{2}\left\|\epsilon_{g}\right\|_{\Gamma_{h}} \cdot\left\|u_{h}^{n+1}-g_{h}\right\|_{\Gamma_{h}} \\
& \leq C_{g} \mu\left\|u_{h}^{n+1}-g_{h}\right\| .
\end{aligned}
$$


Also, from (30), we have

$$
\left|\mathcal{H}\left(u_{h}^{n+1}-u_{h}^{n}, u_{h}^{n+1}-g_{h}\right)\right| \leq \frac{\mu}{h}\left\|u_{h}^{n+1}-u_{h}^{n}\right\| \cdot\left\|u_{h}^{n+1}-g_{h}\right\| .
$$

Putting everything together, we have

$$
\begin{aligned}
& \left\|u_{h}^{n+1}-u_{h}^{n}\right\|^{2}+\left(1-C_{g}(\mu+1) \tau\right)\left\|u_{h}^{n+1}-g_{h}\right\|^{2}-\left\|u_{h}^{n}-g_{h}\right\|^{2} \\
& \leq-\tau \sum_{1 \leq j \leq N}\left[u_{h}^{n+1}-g_{h}\right]^{2}+\frac{\mu \tau}{h}\left\|u_{h}^{n+1}-u_{h}^{n}\right\| \cdot\left\|u_{h}^{n+1}-g_{h}\right\| .
\end{aligned}
$$

Since $\tau \leq C_{1} h^{2}$, we have

$$
\begin{aligned}
& \left\|u_{h}^{n+1}-u_{h}^{n}\right\|^{2}+\left(1-C_{g}(\mu+1) \tau\right)\left\|u_{h}^{n+1}-g_{h}\right\|^{2}-\left\|u_{h}^{n}-g_{h}\right\|^{2} \\
\leq & -\tau \sum_{1 \leq j \leq N}\left[u_{h}^{n+1}-g_{h}\right]^{2}+C_{1} \mu \sqrt{\tau}\left\|u_{h}^{n+1}-u_{h}^{n}\right\| \cdot\left\|u_{h}^{n+1}-g_{h}\right\| \\
\leq & -\tau \sum_{1 \leq j \leq N}\left[u_{h}^{n+1}-g_{h}\right]^{2}+\frac{1}{2}\left\|u_{h}^{n+1}-u_{h}^{n}\right\|^{2}+\frac{1}{2} \mu^{2} C_{1}^{2} \tau\left\|u_{h}^{n+1}-g_{h}\right\|^{2} .
\end{aligned}
$$

Hence, define $C^{\prime}=\frac{1}{2} C_{1}^{2} \mu^{2}+C_{g}(\mu+1)$, we have

$$
\frac{1}{2}\left\|u_{h}^{n+1}-u_{h}^{n}\right\|^{2}+\left(1-C^{\prime} \tau\right)\left\|u_{h}^{n+1}-g_{h}\right\|^{2}-\left\|u_{h}^{n}-g_{h}\right\|^{2} \leq-\tau \sum_{1 \leq j \leq N}\left[u_{h}^{n+1}-g_{h}\right]^{2} .
$$

Finally

$$
\left\|u_{h}^{n+1}-g_{h}\right\| \leq \frac{1}{1-C^{\prime} \tau}\left\|u_{h}^{n}-g_{h}\right\| \leq e^{2 C^{\prime} \tau}\left\|u_{h}^{n}-g_{h}\right\|,
$$

when $\tau$ is small enough, then

$$
\left\|u_{h}^{n}-g_{h}\right\| \leq e^{C_{0} t}\left\|u_{h}^{0}-g_{h}\right\|,
$$

therefore

$$
\left\|u_{h}^{n}\right\| \leq e^{C_{0} t}|| u_{h}^{0}-g_{h}\|+\| g_{h} \|,
$$

where $C_{0}=2 C^{\prime}=C_{1}^{2} \mu^{2}+2 C_{g}(\mu+1)$.

4.2. Stability for the RK2 and RK3 schemes. The stability of the fully discrete DG scheme with RK2 and RK3 has been proved in [22]. Here we use these results of [22] and generalize some of them to the obstacle problems.

Theorem 4.2. For piecewise linear approximation under the $C F L$ condition $\tau \leq C_{1} h$, the RK2 scheme is stable, i.e.

$$
\left\|u_{h}^{n}-g_{h}\right\| \leq e^{C_{0} t}\left\|u_{h}^{0}-g_{h}\right\|
$$

and

$$
\left\|u_{h}^{n}\right\| \leq e^{C_{0} t}\left\|u_{h}^{0}-g_{h}\right\|+\left\|g_{h}\right\|,
$$

where $C_{0}=2 C_{g}(3 \mu+1)\left(2+\mu C_{1}\right)$. 
Proof. Since

$$
u_{h}^{n+1}\left(x_{j}^{\alpha}\right)=\max \left(\tilde{u}_{h}^{n+1}\left(x_{j}^{\alpha}\right), g_{h}\left(x_{j}^{\alpha}\right)\right)
$$

from the quadrature rule, we know that

$$
\left\|u_{h}^{n+1}-g_{h}\right\|^{2}=\sum_{j, \alpha} w_{\alpha}\left(u_{h}^{n+1}-g_{h}\right)^{2}\left(x_{j}^{\alpha}\right) \leq \sum_{j, \alpha} w_{\alpha}\left(\tilde{u}_{h}^{n+1}-g_{h}\right)^{2}\left(x_{j}^{\alpha}\right)=\left\|\tilde{u}_{h}^{n+1}-g_{h}\right\|^{2} .
$$

From the appendix in [22], we know that for piecewise linear approximations $\left\|\tilde{u}_{h}^{n+1}\right\| \leq$ $\left\|u_{h}^{n}\right\|$. Therefore,

$$
\left\|\tilde{u}_{h}^{n+1}-g_{h}\right\|^{2}-\left\|u_{h}^{n}-g_{h}\right\|^{2}+2\left(\tilde{u}_{h}^{n+1}-u_{h}^{n}, g_{h}\right)=\left\|\tilde{u}_{h}^{n+1}\right\|^{2}-\left\|u_{h}^{n}\right\|^{2} \leq 0 .
$$

From the formulation of the RK2 scheme

$$
2\left(\tilde{u}_{h}^{n+1}-u_{h}^{n}, g_{h}\right)=\tau \mathcal{H}\left(u_{h}^{n}, g_{h}\right)+\tau \mathcal{H}\left(u_{h}^{n, 1}, g_{h}\right) .
$$

Hence,

$$
\left\|\tilde{u}_{h}^{n+1}-g_{h}\right\|^{2}-\left\|u_{h}^{n}-g_{h}\right\|^{2} \leq-\tau \mathcal{H}\left(u_{h}^{n}, g_{h}\right)-\tau \mathcal{H}\left(u_{h}^{n, 1}, g_{h}\right) .
$$

On the other hand, we have

$$
\mathcal{H}\left(u_{h}^{n}, g_{h}\right)=\mathcal{H}\left(u_{h}^{n}, g\right)-\mathcal{H}\left(u_{h}^{n}, \epsilon_{g}\right) .
$$

Since $g$ is continuous,

$$
\left|\mathcal{H}\left(u_{h}^{n}, g\right)\right| \leq\left\|u_{h}^{n}\right\| \cdot|| g_{x}\left\|\leq C_{g}\right\| u_{h}^{n} \|,
$$

and furthermore

$$
\left|\mathcal{H}\left(u_{h}^{n}, \epsilon_{g}\right)\right| \leq\left\|u_{h}^{n}\right\| \cdot\left\|\left(\epsilon_{g}\right)_{x}\right\|+\sqrt{2}\left\|u_{h}^{n}\right\|_{\Gamma_{h}} \cdot\left\|\epsilon_{g}\right\|_{\Gamma_{h}} \leq 3 C_{g} \mu\left\|u_{h}^{n}\right\| .
$$

In summary

and similarly

$$
\left|\mathcal{H}\left(u_{h}^{n}, g_{h}\right)\right| \leq C_{g}(3 \mu+1)|| u_{h}^{n}||
$$

Therefore,

$$
\left|\mathcal{H}\left(u_{h}^{n, 1}, g_{h}\right)\right| \leq C_{g}(3 \mu+1)\left\|u_{h}^{n, 1}\right\| .
$$

Since

$$
\begin{aligned}
\left\|u_{h}^{n, 1}\right\|^{2} & =\left(u_{h}^{n, 1}, u_{h}^{n, 1}\right)=\left(u_{h}^{n}, u_{h}^{n, 1}\right)+\tau \mathcal{H}\left(u_{h}^{n}, u_{h}^{n, 1}\right) \\
& \leq\left\|u_{h}^{n}\right\| \cdot\left\|u_{h}^{n, 1}\right\|+\mu \frac{\tau}{h}\left\|u_{h}^{n}\right\| \cdot\left\|u_{h}^{n, 1}\right\| \leq\left(1+\mu C_{1}\right)\left\|u_{h}^{n}\right\| \cdot\left\|u_{h}^{n, 1}\right\|,
\end{aligned}
$$

we have

$$
\left\|u_{h}^{n, 1}\right\| \leq\left(1+\mu C_{1}\right)\left\|u_{h}^{n}\right\| .
$$

We deduce from (34) and (35) the following bound

$$
\begin{aligned}
\left\|\tilde{u}_{h}^{n+1}-g_{h}\right\|^{2}-\left\|u_{h}^{n}-g_{h}\right\|^{2} & \leq C^{\prime} \tau\left\|u_{h}^{n}\right\| \\
& \leq C^{\prime} \tau\left\|u_{h}^{n}-g_{h}\right\|+C^{\prime} \tau\left\|g_{h}\right\| \leq C^{\prime} \tau\left\|u_{h}^{n}-g_{h}\right\|+C^{\prime} C_{g} \tau,
\end{aligned}
$$

where $C^{\prime}=C_{g}(3 \mu+1)\left(2+\mu C_{1}\right)$. Now, we can see

$$
\left\|u_{h}^{n+1}-g_{h}\right\|^{2} \leq\left\|\tilde{u}_{h}^{n+1}-g_{h}\right\|^{2} \leq\left(1+C^{\prime} \tau\right)\left\|u_{h}^{n}-g_{h}\right\|^{2}+C^{\prime} C_{g} \tau,
$$


and from here, we deduce

$$
\left\|u_{h}^{n}-g_{h}\right\| \leq e^{C_{0} t}\left\|u_{h}^{0}-g_{h}\right\|,
$$

where $C_{0}=2 C^{\prime}=2 C_{g}(3 \mu+1)\left(2+\mu C_{1}\right)$ and the scheme is stable.

Theorem 4.3. Under the CFL condition $\tau \leq C_{1} h$, the RK3 scheme is stable, i.e.

$$
\left\|u_{h}^{n}-g_{h}\right\| \leq e^{C_{0} t}\left\|u_{h}^{0}-g_{h}\right\|,
$$

and

$$
\left\|u_{h}^{n}\right\| \leq e^{C_{0} t}|| u_{h}^{0}-g_{h}\|+\| g_{h} \|,
$$

where $C_{0}=2 C^{\prime}=\frac{2}{3} C_{g}(3 \mu+1)\left(6+3 \mu C_{1}+\mu^{2} C_{1}^{2}\right)$.

Proof. The proof follows the same line as in the proof of the previous theorem. The only difference is that the scheme from RK3 yields

$$
2\left(\tilde{u}_{h}^{n+1}-u_{h}^{n}, g_{h}\right)=\frac{1}{3} \tau\left(\mathcal{H}\left(u_{h}^{n}, g_{h}\right)+\mathcal{H}\left(u_{h}^{n, 1}, g_{h}\right)+4 \mathcal{H}\left(u_{h}^{n, 2}, g_{h}\right)\right) .
$$

From similar arguments as in the previous proof,

$$
\left|2\left(\tilde{u}_{h}^{n+1}-u_{h}^{n}, g_{h}\right)\right| \leq \frac{1}{3} C_{g}(3 \mu+1) \tau\left(|| u_{h}^{n}\|+\| u_{h}^{n, 1}\|+4\| u_{h}^{n, 2}||\right) .
$$

In the proof of the previous theorem, we have already proved $\left\|u_{h}^{n, 1}\right\| \leq\left(1+\mu C_{1}\right)\left\|u_{h}^{n}\right\|$. Actually a similar conclusion holds for $\left\|u_{h}^{n, 2}\right\|$ as well.

Since

$$
\begin{aligned}
& \left\|u_{h}^{n, 2}\right\|^{2}=\left(u_{h}^{n, 2}, u_{h}^{n, 2}\right)=\frac{3}{4}\left(u_{h}^{n}, u_{h}^{n, 2}\right)+\frac{1}{4}\left(u_{h}^{n, 1}, u_{h}^{n, 2}\right)+\frac{1}{4} \tau \mathcal{H}\left(u_{h}^{n, 1}, u_{h}^{n, 2}\right) \\
& \leq \frac{3}{4}\left\|u_{h}^{n}\right\| \cdot\left\|u_{h}^{n, 2}\right\|+\frac{1}{4}\left\|u_{h}^{n, 1}\right\| \cdot\left\|u_{h}^{n, 2}\right\|+\mu \frac{\tau}{4 h}\left\|u_{h}^{n, 1}\right\| \cdot\left\|u_{h}^{n, 2}\right\| \\
& \leq \frac{1}{4}\left(3+\left(1+\mu C_{1}\right)^{2}\right)\left\|u_{h}^{n}\right\| \cdot\left\|u_{h}^{n, 2}\right\|,
\end{aligned}
$$

we have $\left\|u_{h}^{n, 2}\right\| \leq \frac{1}{4}\left(3+\left(1+\mu C_{1}\right)^{2}\right)\left\|u_{h}^{n}\right\|$. Therefore,

$$
\left|2\left(\tilde{u}_{h}^{n+1}-u_{h}^{n}, g_{h}\right)\right| \leq C^{\prime} \tau|| u_{h}^{n} \|,
$$

where $C^{\prime}=\frac{1}{3} C_{g}(3 \mu+1)\left(6+3 \mu C_{1}+\mu^{2} C_{1}^{2}\right)$. Then following the same argument of the previous theorem, we have

$$
\left\|u_{h}^{n}-g_{h}\right\| \leq e^{C_{0} t}\left\|u_{h}^{0}-g_{h}\right\|,
$$

where $C_{0}=2 C^{\prime}=\frac{2}{3} C_{g}(3 \mu+1)\left(6+3 \mu C_{1}+\mu^{2} C_{1}^{2}\right)$ and the scheme is stable.

\section{Numerical RESUlts}

In this section, we consider various numerical tests to validate our scheme. In one dimensional and two dimensional setting, we perform accuracy test for the solution away from singular points. For two dimensional examples, we are mainly concerned with evolution of the zero level set. 
Example 1 (1-d, linear). We first consider a one-dimensional test:

$$
\begin{aligned}
& \min \left(u_{t}+u_{x}, u-g(x)\right)=0, \quad t>0, x \in[-1,1], \\
& u(0, x)=u_{0}(x), \quad x \in[-1,1],
\end{aligned}
$$

with periodic boundary conditions and $g(x):=\sin (\pi x), u_{0}(x):=0.5+\sin (\pi x)$. In that case, for times $0 \leq t \leq 1$, the exact solution is given by :

$$
u^{(1)}(t, x)= \begin{cases}\max \left(u_{0}(x-t), g(x)\right) & \text { if } t<\frac{1}{3}, \\ \max \left(u_{0}(x-t), g(x), 1_{x \in[0.5,1]}\right) & \text { if } t \in\left[\frac{1}{3}, \frac{1}{3}+\frac{1}{2}\right], \\ \max \left(u_{0}(x-t), g(x), 1_{x \in\left[-1, t-\frac{1}{3}-\frac{1}{2}\right] \cup[0.5,1]}\right) & \text { if } t \in\left[\frac{1}{3}+\frac{1}{2}, 1\right] .\end{cases}
$$

In Table 1 we show the numerical errors away from the singular points of the solution $u(t,$.$) : we have computed all error norms in the region [-1,1] \backslash\left(\bigcup_{i=1,3}\left[s_{i}-\delta, s_{i}+\delta\right]\right)$ where $s_{1}:=-0.1349733, s_{2}:=0.5$ and $s_{3}=2 / 3$ are the three singular points of the solution at time $t=0.5$, and with $\delta=0.1$. We observe the optimal third order convergence rate for $P^{2}$ elements.

TABle 1. Example 1. $t=0.5 . P^{2}$ elements.

\begin{tabular}{rl|lc|cc|cc}
$N_{x}$ & $\Delta x$ & $L^{1}$-error & order & $L^{2}$-error & order & $L^{\infty}$-error & order \\
\hline \hline 40 & $5.00 \mathrm{e}-2$ & $3.34 \mathrm{e}-05$ & 2.41 & $1.01 \mathrm{e}-04$ & 1.98 & $7.02 \mathrm{e}-04$ & 2.20 \\
80 & $2.50 \mathrm{e}-2$ & $1.77 \mathrm{e}-06$ & 4.24 & $3.64 \mathrm{e}-06$ & 4.79 & $2.82 \mathrm{e}-05$ & 4.64 \\
160 & $1.25 \mathrm{e}-2$ & $1.78 \mathrm{e}-07$ & 3.31 & $2.91 \mathrm{e}-07$ & 3.64 & $2.40 \mathrm{e}-06$ & 3.55 \\
320 & $6.25 \mathrm{e}-3$ & $2.13 \mathrm{e}-08$ & 3.06 & $3.43 \mathrm{e}-08$ & 3.08 & $1.28 \mathrm{e}-07$ & 4.23 \\
640 & $3.13 \mathrm{e}-3$ & $2.66 \mathrm{e}-09$ & 3.00 & $4.28 \mathrm{e}-09$ & 3.00 & $1.60 \mathrm{e}-08$ & 3.00 \\
1280 & $1.56 \mathrm{e}-3$ & $3.32 \mathrm{e}-10$ & 3.00 & $5.35 \mathrm{e}-10$ & 3.00 & $2.00 \mathrm{e}-09$ & 3.00
\end{tabular}

In Figure 1 we show the numerical solution which agrees well with the exact solution everywhere.
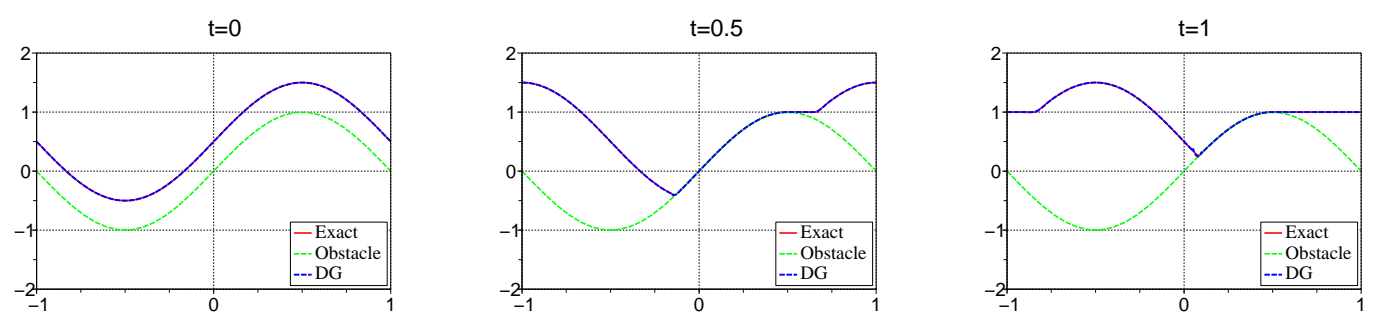

Figure 1. Example 1, times $t=0$ (initial data), $t=0.5$ and $t=1$, using $P^{2}$ elements with $N_{x}=20$ mesh cells (obstacle : green dotted line) 
Example 2 (1-d, nonlinear $)$. We consider a one-dimensional test with a nonlinear Hamiltonian:

$$
\begin{aligned}
& \min \left(u_{t}+\left|u_{x}\right|, u-g(x)\right)=0, \quad t>0, x \in[-1,1], \\
& u(0, x)=u_{0}(x), \quad x \in \Omega,
\end{aligned}
$$

with periodic boundary conditions and $g(x):=\sin (\pi x), u_{0}(x):=0.5+\sin (\pi x)$. In this particular case, the exact solution is given by:

$$
u(t, x)=\max (\bar{u}(t, x), g(x))
$$

where $\bar{u}$ is the solution of the Eikonal equation $u_{t}+\left|u_{x}\right|=0$ and can be computed from the formula $\bar{u}(t, x):=\min _{y \in[x-t, x+t]} u_{0}(y)$ and which simplifies here to

$$
\bar{u}(t, x)= \begin{cases}u^{0}(x+t) & \text { if } x \leq-0.5-t \\ -0.5 & \text { if } x \in[-0.5-t,-0.5+t] \\ \min \left(u^{0}(x-t), u^{0}(x+t)\right) & \text { if } x \geq-0.5+t\end{cases}
$$

For times $t \geq \frac{1}{3}$, the solution remains unchanged.

In Figure 2 we show the numerical solution for times $t \in\{0.2,0.4\}$, which agrees well with the exact solution.
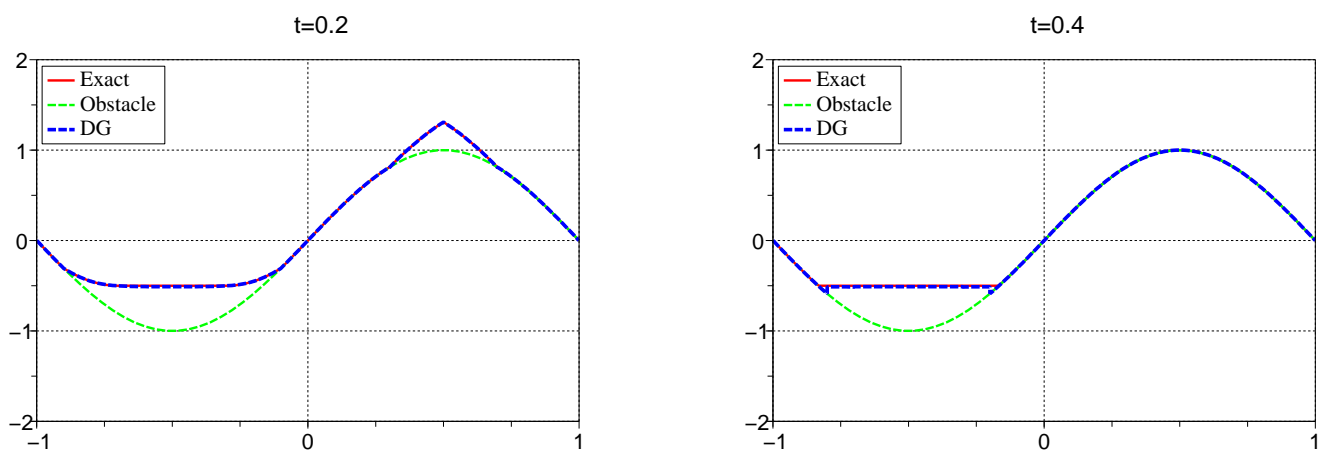

Figure 2. Example 2, numerical and exact solutions at times $t=0.2$ and $t=0.4, N_{x}=20$, using $P^{2}$ (obstacle : green dotted line).

Example 3 (2-d, linear, accuracy test). The equation solved is

$$
\begin{aligned}
& \min \left(u_{t}+\frac{1}{2} u_{x}+\frac{1}{2} u_{y}, u-g(x, y)\right)=0, \quad t>0, \quad(x, y) \in \Omega, \\
& u(0, x, y)=u_{0}(x, y), \quad(x, y) \in \Omega,
\end{aligned}
$$

where $g(x, y):=\sin (\pi(x+y)), u_{0}(x, y)=0.5+g(x, y)$, and $\Omega=[-1,1]^{2}$ with periodic boundary conditions. The exact solution is known and is obtained as in Example 1:

$$
u(t, x, y)=u^{(1)}(t, x+y)
$$

(where $u^{(1)}$ is the exact solution for Example 1).

Accuracy results are shown in Table 2 for time $t=0.5$, The errors are computed away from the singular zone, that is, only in the region $\left\{(x, y) \in \Omega, 1 \leq i \leq 3, d\left(x+y-s_{i}, 2 \mathbb{Z}\right) \geq\right.$ $\delta)\}$, with $\delta=0.1$. We observe optimal convergence rate in this example. 
TABle 2. Example 3. $t=0.5 . Q^{2}$ elements.

\begin{tabular}{cc|cc|cc|cc}
$N_{x}=N_{y}$ & $\Delta x=\Delta y$ & $L^{1}$-error & order & $L^{2}$-error & order & $L^{\infty}$-error & order \\
\hline \hline 10 & $2.00 \mathrm{e}-1$ & $7.70 \mathrm{e}-03$ & - & $1.03 \mathrm{e}-02$ & - & $1.04 \mathrm{e}-01$ & - \\
20 & $1.00 \mathrm{e}-1$ & $9.27 \mathrm{e}-04$ & 3.05 & $1.28 \mathrm{e}-03$ & 3.01 & $8.71 \mathrm{e}-03$ & 3.58 \\
40 & $5.00 \mathrm{e}-2$ & $9.48 \mathrm{e}-05$ & 3.29 & $1.67 \mathrm{e}-04$ & 2.94 & $1.04 \mathrm{e}-03$ & 3.06 \\
80 & $2.50 \mathrm{e}-2$ & $7.15 \mathrm{e}-06$ & 3.73 & $1.11 \mathrm{e}-05$ & 3.91 & $1.02 \mathrm{e}-04$ & 3.34
\end{tabular}

Example $4(\mathbf{2}-\mathbf{d}$, linear $)$. The initial data is $u_{0}(\mathbf{x}):=\|\mathbf{x}-(-0.5,0)\|_{\infty}-0.3$, with $\mathbf{x}=(x, y)$. The obstacle is coded by $g(\mathbf{x}):=0.25-\|\mathbf{x}-(0,0.25)\|_{\infty}$. The equation solved is

$$
\begin{aligned}
& \min \left(u_{t}+u_{x}, u-g(x, y)\right)=0, \quad t>0, \quad(x, y) \in \Omega, \\
& u(0, x, y)=u_{0}(x, y), \quad(x, y) \in \Omega,
\end{aligned}
$$

and the domain is $\Omega:=[-1,1]^{2}$ (with periodic boundary conditions).

Results are shown in Fig. 3 at three different times, which agree well with the exact solution.
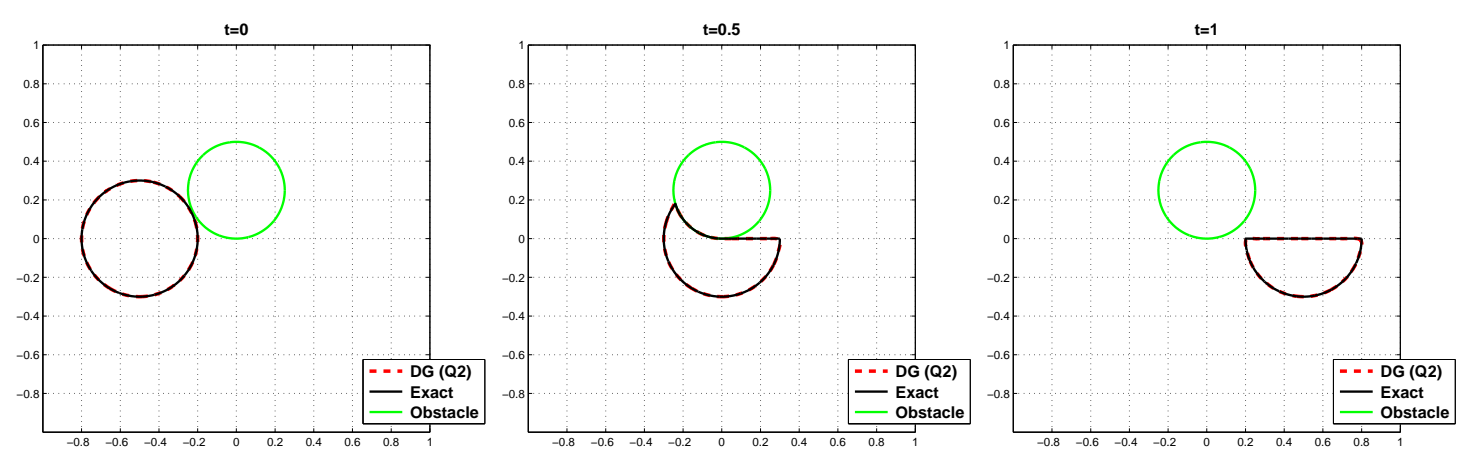

Figure 3. Example $4\left(N_{x}=N_{y}=40\right)$, times $t \in\{0,0.5,1\}$

Example 5 (2-d, linear, variable coefficients). We consider

$$
f(x, y):=\left(\begin{array}{r}
-2 \pi y \\
2 \pi x
\end{array}\right) \max \left(1-\|\mathbf{x}\|_{2}, 0\right) .
$$

where $\|\mathbf{x}\|_{2}:=\sqrt{x^{2}+y^{2}}$ and with a Lipschitz continuous initial data $u_{0}$ :

$$
u_{0}(x, y)=\min (\max (y,-1), 1) .
$$

The function $u_{0}$ has a 0 -level set which is the $x$ axis: $\left\{\mathbf{x}=(x, y) \in \mathbb{R}^{2} \mid y=0\right\}$. When there is no obstacle function, the exact solution is known ${ }^{1}$

Computations have been done up to time $t=1.0$ (see Figure 4), on the domain $(x, y) \in$ $[-1,1]^{2}$, on a $20 \times 20$ mesh, with piecewise $Q^{2}$ elements in space. The obstacle constraint is well taken into account (the square obstacle prevents the front to evolve between two arcs tangent to the square).

$$
{ }^{1} u(t, \mathbf{x}):=u_{0}(R(-2 \pi a(\mathbf{x}) t) \mathbf{x}) \text { where } R(\theta):=\left(\begin{array}{rr}
\cos (\theta) & -\sin (\theta) \\
\sin (\theta) & \cos (\theta)
\end{array}\right) \text { and } a(\mathbf{x}):=\max \left(1-\|\mathbf{x}\|_{2}, 0\right) .
$$



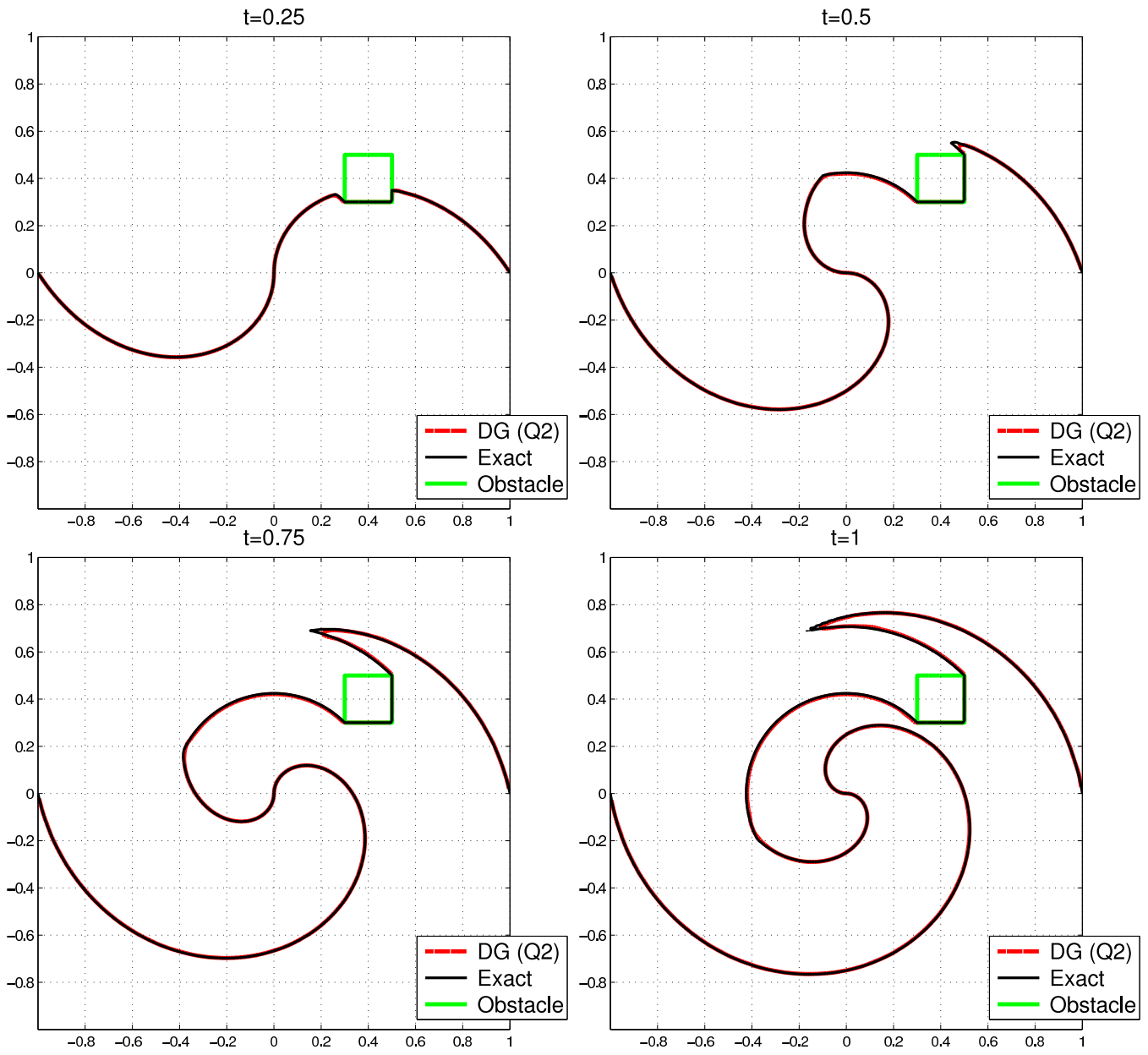

Figure 4. Example 5, plots at times $t \in\{0.25,0.5,0.75,1\}$, with $Q^{2}$ and $40 \times 40$ mesh cells.

Example 6 (2-d, nonlinear). In this example we consider an initial data $u_{0}(x, y):=$ $\|(x, y)-(1,0)\|_{\infty}-0.5$, an obstacle coded by $g(x, y):=0.5-\|(x, y)-(0,0.5)\|_{\infty}$, and the problem

$$
\begin{aligned}
& \min \left(u_{t}+\max (0,2 \pi(-y, x) \cdot \nabla u), u-g(x, y)\right)=0, \quad t>0, \quad(x, y) \in \Omega, \\
& u(0, x, y)=u_{0}(x, y), \quad(x, y) \in \Omega,
\end{aligned}
$$

The domain is $\Omega:=[-2,2]^{2}$. Thus we want to compute the backward reachable set associated to the dynamics $f(x, y)=-2 \pi(-y, x)$ and the target $\mathcal{T}=\left\{(x, y), u_{0}(x, y) \leq 0\right\}$, together with an obstacle or forbidden zone represented by $\{(x, y), g(x, y) \geq 0\}$.

Result is shown in Fig. 5 at different times. No entropy fix was needed, and we see a very good agreement with the exact solution.

Remark 5.1 (Comparison with a penalization approach). We consider this nonlinear example to compare with the penalization approach. Here we have numerically approximated equation (9) by the DG method. We have tested various penalization parameters 

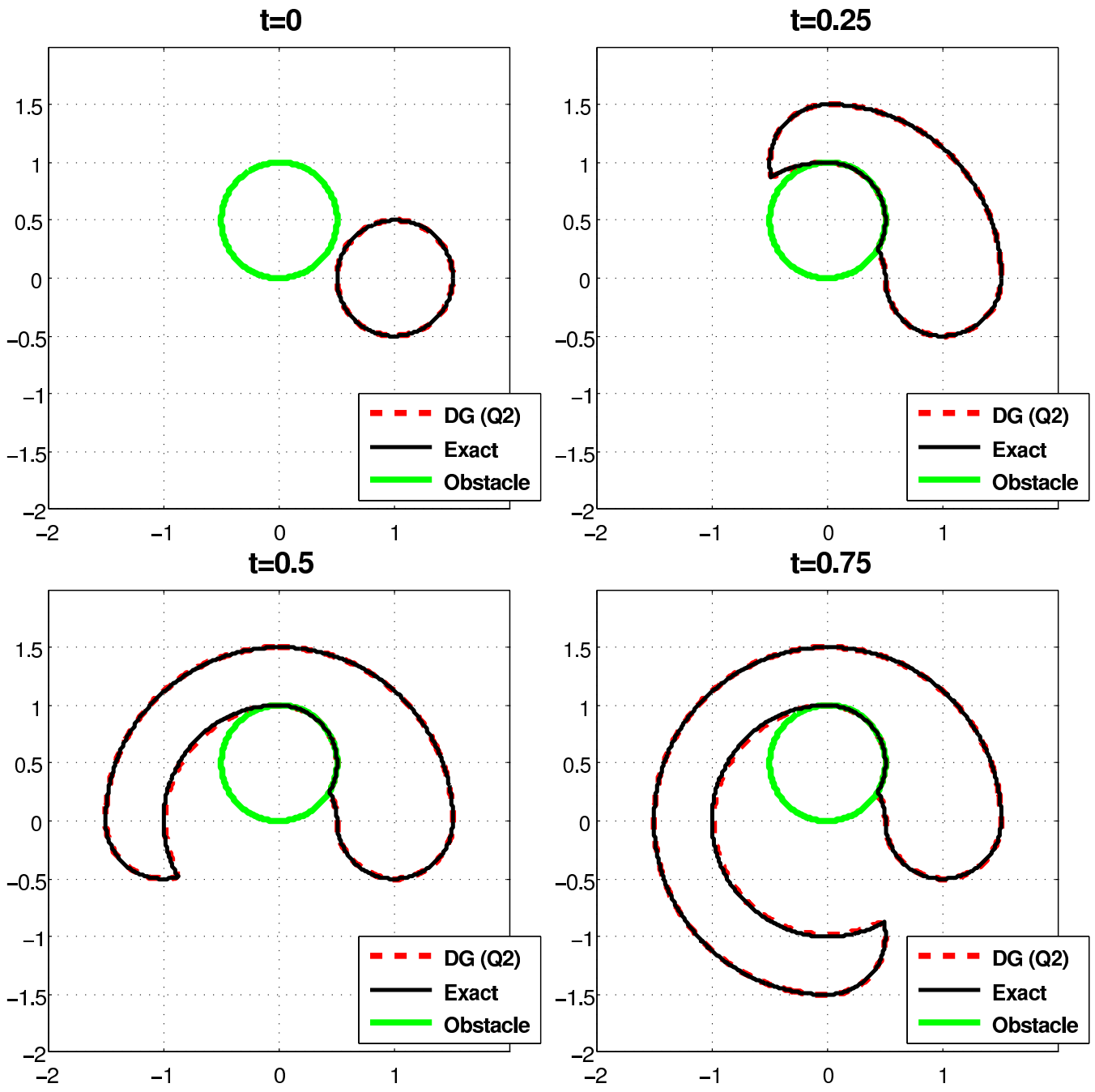

Figure 5. Example 6, plots at times $t \in\{0,0.25,0.5,0.75\}$, with $Q^{2}$ and $80 \times 80$ mesh cells.

$\varepsilon \in\left\{10^{-k}, k=1, \ldots, 5\right\}$. Results are shown in Fig. 6 in the case of $\varepsilon=10^{-2}$. Although the qualitative behavior of the zero-level set is obtained, we see that we lose completely the precision of the DG method.

There is no intuitive way to define $\varepsilon$ and we show here the results for the penalization parameter that gave the best results. If $\varepsilon$ is greater than 0.01 then the obstacle is not well enough taken into account. On the other hand if $\varepsilon$ is too small then the solution becomes too large in the obstacle domain (where $g(x, y)>0$ ) and the 0-level set of the numerical solution becomes badly perturbed. 

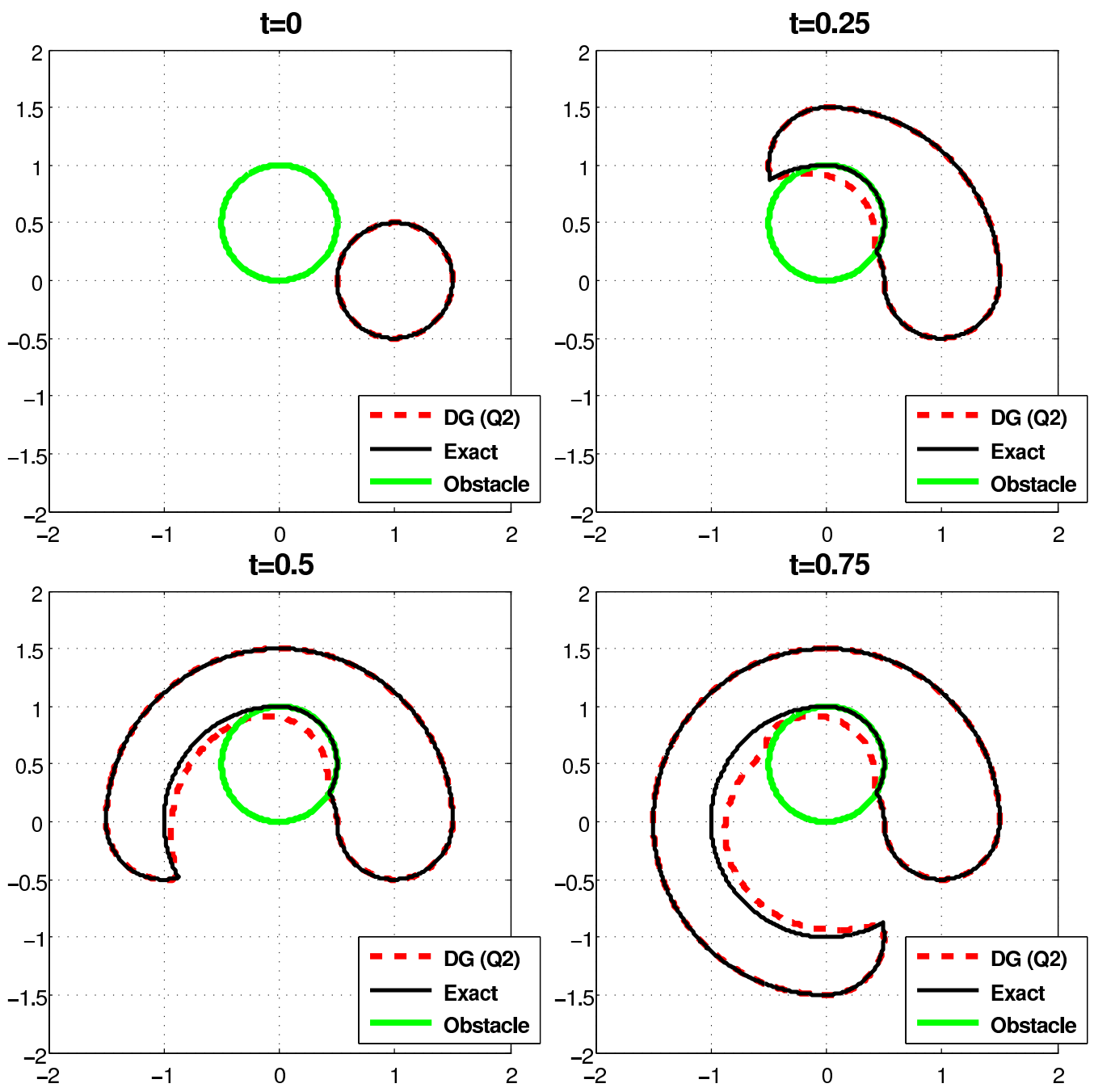

Figure 6. Example 6: Penalization approach with penalization parameter $\varepsilon=10^{-2}, t \in\{0,0.25,0.5,0.75\}$, with $Q^{2}$ and $80 \times 80$ cells

Example 7. We consider the problem

$$
\begin{aligned}
& \min \left(u_{t}+\max \left(0, u_{x}+\frac{1}{2}\left|u_{y}\right|\right), u-g(x, y)\right)=0, \quad t>0, \quad(x, y) \in \Omega, \\
& u(0, x, y)=u_{0}(x, y), \quad x \in \Omega,
\end{aligned}
$$

with $u_{0}(\mathbf{x}):=\|\mathbf{x}-(-1.0,0)\|_{\infty}-0.5$ and $g(\mathbf{x}):=\min \left(0.25,\|\mathbf{x}-(0.2,0)\|_{2}-0.5\right)$, corresponding to a square initial data and a disk obstacle.

In this example the "entropy fix" is needed. Results are given in Fig. 7. The numerical solution again agrees well with the exact solution. 

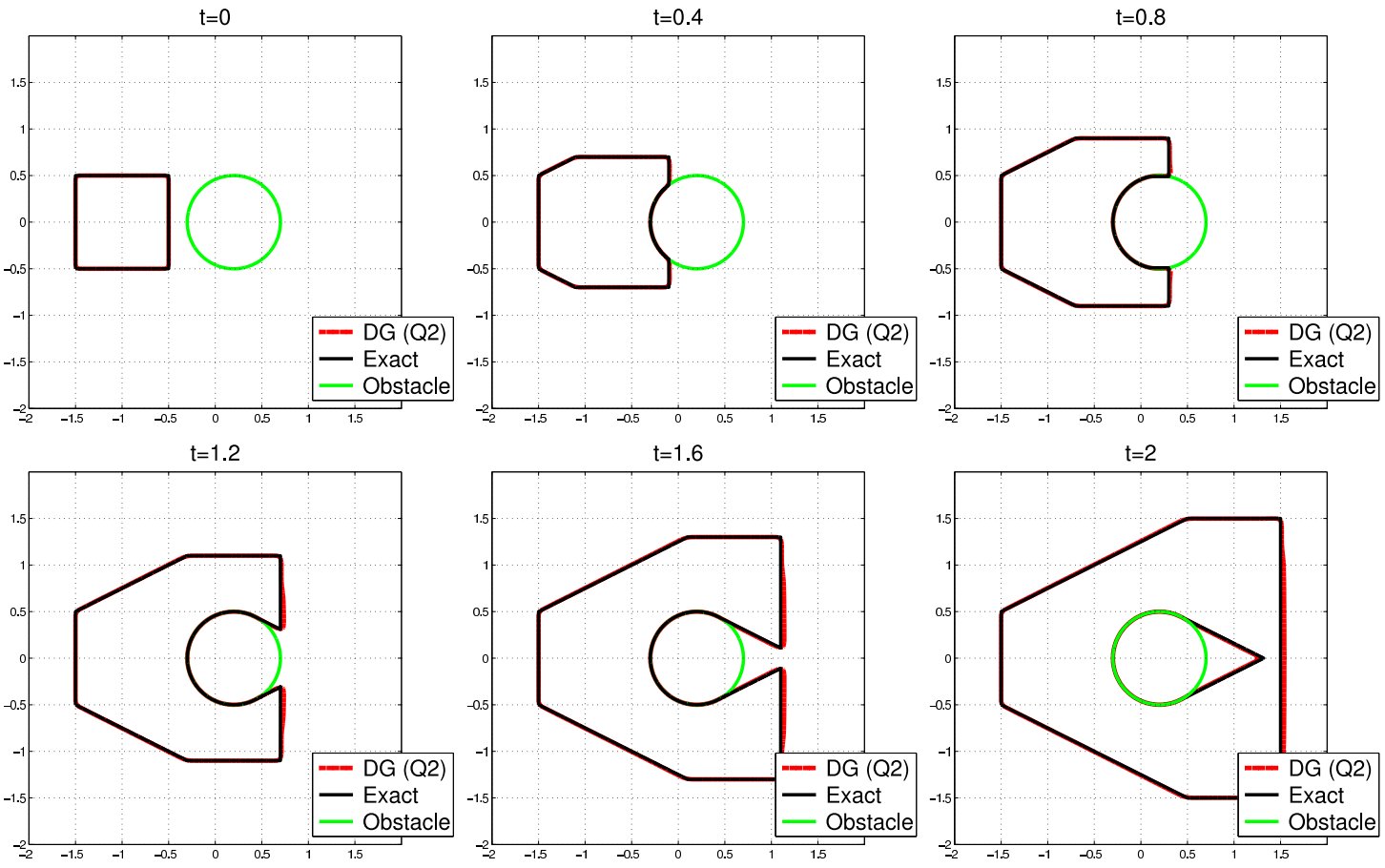

Figure 7. Example 7, plots at times $t \in\{0,0.4,0.8,1.2,1.6,2.0\}$, with $Q_{2}$ and $80 \times 80$ mesh cells.

Example 8 (Narrow band approach). In this example we propose a simple narrow band approach adapted to front propagation problems using the DG schemes of the present paper.

The narrow band algorithm is as follows. We define a "cutoff" value, typically

$$
C:=2 \Delta x \text {. }
$$

- The initial data $u_{0}$ is transformed into

$$
\tilde{u}_{0}(x, y):=\min \left(C, \max \left(-C, u_{0}(x, y)\right)\right) .
$$

- At each time step, (i) for all index $i, j$ (mesh cell centered at $\left(x_{i}, y_{j}\right)$ ), we compute

$$
n \log o_{i, j}^{0}= \begin{cases}1 & \text { if }\left|u^{n}\left(x_{i}, y_{j}\right)\right| \leq 0.99 C, \\ 0 & \text { otherwise }\end{cases}
$$

(where $u^{n}\left(x_{i}, y_{j}\right)$ denotes the $D G$ polynomial value at the mesh cell center and at time $\left.t_{n}\right)$.

- (ii) for all index $i, j$, we compute

$$
n \log o_{i, j}:=\max \left(n \log o_{i, j}^{0}, n \log o_{i, j+1}^{0}, n \log o_{i, j-1}^{0}, n \log o_{i+1, j}^{0}, n \log o_{i-1, j}^{0}\right)
$$

- (iii) finally we do the DG computations only on cells $(i, j)$ such that $n \log o_{i, j}=1$.

In Figure 8 we apply the narrow band approach to the obstacle problem of example 6 . We have plotted with dots the narrow band cells which are used at different times. 
Furthermore in Table 3 we show some CPU times for a simplified advection problem (rotation of a circle)

$$
\begin{aligned}
& u_{t}+2 \pi(-y, x) \cdot \nabla u=0, \quad t>0, \quad(x, y) \in \Omega, \\
& u(0, x, y)=u_{0}(x, y), \quad(x, y) \in \Omega,
\end{aligned}
$$

and same initial data $u_{0}$ as in Example 6. (The results for the complete Example 6 are similar excepted for a scaling factor on the CPU times.)

Here the "order" is computed as the ratio of CPU times time $\left(N_{x}\right) / \operatorname{time}\left(N_{x} / 2\right)$. For large $N_{x}$ values, we observe an order of 8 (approximately) for the full approach, and of 4 (approximately) for the narrow band approach. This is justified for the narrow band approach because it will use a number of cells proportional to the length of the front (which accounts for a factor of 2) and there is another factor of 2 coming from the CFL condition $\tau \leq$ const. $\Delta x$.

Note that in Table 3 we have made used of a parallel version of the code (Fortran OpenMP on a 8 core processor) to reduce the computational cost, since the DG schemes have the advantage to be easily parallelizable.

TABLE 3. (Example 8) comparison of CPU times (in sec.) for full and narrow band approaches for $(50), t=0.5$

\begin{tabular}{r|rc|rc|c}
$N_{x}$ & full & "order" & narrow band & "order" & Gain (full / band) \\
\hline \hline 20 & $8.1 \mathrm{~s}$ & - & $6.9 \mathrm{~s}$ & - & 1.17 \\
40 & $45.2 \mathrm{~s}$ & 5.58 & $17.1 \mathrm{~s}$ & 2.47 & 2.64 \\
80 & $347.4 \mathrm{~s}$ & 7.68 & $83.4 \mathrm{~s}$ & 4.87 & 4.16 \\
160 & $2705.3 \mathrm{~s}$ & 7.78 & $386.0 \mathrm{~s}$ & 4.62 & 7.00
\end{tabular}
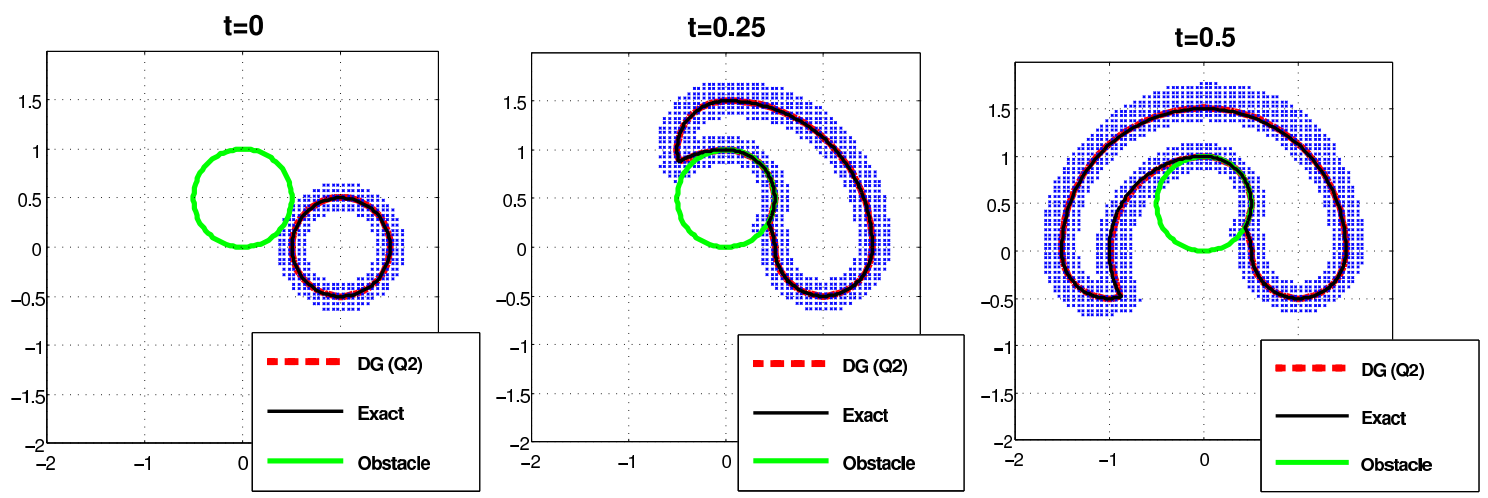

Figure 8. (Example 8) Narrow band approach for same problem as in example 6 , with $t \in\{0,0.25,0.5\}$, with $Q_{2}$ and $80 \times 80$ mesh cells.

\section{Concluding Remarks}

In this paper, we propose a DG approach to solve front propagation problems with obstacles. The scheme is explicit in time, and the obstacles are well taken into account 
through a simple projection step. The method is demonstrated to be high order accurate in smooth regions and has good resolution of singularities. Stability analysis are provided for three typical time discretization. Future work includes a thorough investigation of the narrow band approach and applications to higher dimensional problems.

\section{REFERENCES}

[1] Y. Achdou and O. Pironneau. Computational methods for option pricing. Frontiers in Applied Mathematics. SIAM, 2005.

[2] M. Bardi and I. Capuzzo-Dolcetta. Optimal control and viscosity solutions of Hamilton-Jacobi-Bellman equations. Systems and Control: Foundations and Applications. Birkhäuser, Boston, 1997.

[3] E. N. Barron. Viscosity solutions and analysis in $L^{\infty}$. Proceedings of the NATO advanced Study Institute, pages 1 - 60, 1999.

[4] A. Bensoussan and J. L. Lions. C. R. Acad. Sc. Paris, 276(Série A):1411-1415, 1973.

[5] A. Bensoussan and J. L. Lions. On the support of the solution of some variational inequalities of evolution. J. Math. Soc. Japan, 28(1):1-17, 1976.

[6] O. Bokanowski, Y. Cheng, and C.-W. Shu. A discontinuous Galerkin solver for front propagation. Preprint, 2009. Submitted to SIAM J. Sci. Comput.

[7] O. Bokanowski, N. Forcadel, and H. Zidani. Deterministic state constrained optimal control problems without controllability assumptions. To appear in COCV, 2010.

[8] O. Bokanowski, N. Forcadel, and H. Zidani. Reachability and minimal times for state constrained nonlinear problems without any controllability assumption. SIAM J. Control Optim., 48(7):42924316, 2010.

[9] O. Bokanowski, S. Martin, R. Munos, and H. Zidani. An anti-diffusive scheme for viability problems. Appl. Numer. Math., 56(Issue 9, in Numerical Methods for Viscosity Solutions and Applications):11351254, 2006.

[10] P. Cardaliaguet, M. Quincampoix, and P. Saint-Pierre. Optimal times for constrained nonlinear control problems without local controllability. Appl. Math. Optim, 36:21-42, 1997.

[11] P. Cardaliaguet, M. Quincampoix, and P. Saint-Pierre. Numerical schemes for discontinuous value function of optimal control. Set-Valued Analysis, 8:111-126, 2000.

[12] Y. Cheng and C.-W. Shu. A discontinuous Galerkin finite element method for directly solving the Hamilton-Jacobi equations. J. Comput. Phys., 223:398-415, 2007.

[13] B. Cockburn and C.-W. Shu. Runge-Kutta discontinuous Galerkin methods for convection-dominated problems. J. Sci. Comput., 16:173-261, 2001.

[14] H. Frankowska and S. Plaskacz. Semicontinuous solutions of Hamilton-Jacobi-Bellman equations with degenerate state constraints. J. Math. Anal. Appl., 251(2):818-838, 2000.

[15] C. Hu and C.-W. Shu. A discontinuous Galerkin finite element method for Hamilton-Jacobi equations. SIAM J. Sci. Comput., 21:666-690, 1999.

[16] A. Kurzhanski and P. Varayiya. Ellipsoidal techniques for reachability under state constraints. SIAM J. Control Optim., 45:1369-1394, 2006.

[17] F. Li and C.-W. Shu. Reinterpretation and simplified implementation of a discontinuous Galerkin method for Hamilton-Jacobi equations. Appl. Math. Lett., 18:1204-1209, 2005.

[18] F. Li and S. Yakovlev. A central discontinuous Galerkin method for Hamilton-Jacobi equations. J. Sci. Comput., 45:404-428, 2010.

[19] P. Saint-Pierre. Approximation of the viability kernel. Appl. Math. Optim, 29:187-2009, 1994.

[20] H. M. Soner. Optimal control with state-space constraint. II. SIAM J. Control Optim., 24(6):11101122, 1986.

[21] J. Yan and S. Osher. A local discontinuous Galerkin method for directly solving Hamilton-Jacobi equations. J. Comput. Phys., 230:232-244, 2011.

[22] Q. Zhang and C.-W. Shu. Stability analysis and a priori error estimates to the third order explicit runge-kutta discontinuous galerkin method for scalar conservation laws. SIAM J. Numer. Anal., 48:1038-1063, 2010. 
Laboratoire Jacques-Louis Lions, Université Pierre et Marie Curie 75252 Paris Cedex 05 FRANCE

UfR de Mathématiques, Site Chevaleret, Université Paris-Diderot, 75205 Paris Cedex FRANCE

E-mail address: boka@math.jussieu.fr

Department of Mathematics and ICES, University of Texas, Austin, TX 78712, UsA

E-mail address: ycheng@math.utexas.edu

Division of Applied Mathematics, Brown University, Providence, Ri 02912, USA

E-mail address: shu@dam.brown.edu 